INRA Prod. Anim.,

2012, 25 (4), 323-336

\title{
Restreindre l'ingestion du jeune lapin : de nouvelles stratégies pour renforcer sa santé digestive et améliorer son efficacité alimentaire
}

\author{
T. GIDENNE 1,2,3, L. FORTUN-LAMOTHE 1,2,3, S. COMBES $S^{1,2,3}$ \\ ${ }^{1}$ INRA, UMR1289 Tissus Animaux Nutrition Digestion Ecosystème et Métabolisme, \\ F-31326 Castanet-Tolosan, France \\ 2 Université de Toulouse INPT ENSAT, UMR Tissus Animaux Nutrition Digestion Ecosystème et Métabolisme, \\ F-31326 Castanet-Tolosan, France \\ ${ }^{3}$ Université de Toulouse INPT ENVT, UMR Tissus Animaux Nutrition Digestion Ecosystème et Métabolisme, \\ F-31076 Toulouse, France \\ Courriel : thierry.gidenne@toulouse.inra.fr
}

Depuis 2002, l'INRA a conduit un ensemble d'études sur l'impact d'une limitation de l'ingestion sur la santé du jeune lapin. Ce travail a été réalisé en partenariat avec l'ITAVI et l'ensemble des firmes services françaises d'alimentation animale au sein du «Groupe d'Expérimentation Cunicole». Cet article fait le point sur les effets zootechniques et physiologiques des stratégies de restriction alimentaire chez le lapin en croissance'.

La période du sevrage, particulièrement importante pour établir la future croissance de l'animal, est une phase de forte sensibilité aux troubles digestifs chez le jeune mammifère, tels le porcelet, le veau, et le jeune lapin entre 4 et 9 semaines d'âge. Ainsi, les pathologies digestives infectieuses sont la principale cause de mortalité post-sevrage chez le lapin (Marlier et al 2003). Elles peuvent avoir une origine multifactorielle, avec une combinaison d'un ou de plusieurs agents pathogènes (bactéries, coccidies...). En outre, depuis la première épidémie d'Entéropathie Epizootique du Lapin (EEL) en 1997, le contrôle des maladies digestives autour du sevrage est devenu plus complexe en cuniculture. Actuellement, les prescriptions vétérinaires ont souvent recours aux antibiotiques, en approche métaphylactique (voire préventive) pour contrôler l'EEL ou lutter contre les colibacilles entéropathogènes (EPEC). Face à une consommation élevée de médicaments vétérinaires (Chevance et Moulin 2009), il y a donc un besoin urgent de trouver des solutions alternatives et préventives visant à améliorer le contrôle de ces maladies digestives. Diverses améliorations portant sur l'hygiène du logement, l'élevage en bandes, les recommenda- tions nutritionnelles et alimentaires ont été proposées pour le lapin en croissance au cours des dix dernières années. Des études récentes ont précisé les besoins minimaux en fibres pour le lapin en croissance, afin de réduire les risques d'entéropathies spécifiques (EEL, EPEC) ou non spécifiques (Gidenne 2003, Carabaño et al 2008, Gidenne et al 2010).

La restriction temporaire de l'ingestion a été initialement étudiée chez le jeune lapin pour en analyser les effets sur sa croissance et son efficacité digestive (Lebas et Delaveau 1975, Lebas 1979). Toutefois en 2003, le réseau français d'unités expérimentales cunicoles (GEC) a montré pour la première fois, l'impact favorable de la limitation de l'ingestion après le sevrage sur la santé digestive du lapin en croissance (Gidenne et al 2003). D'autres études ont depuis précisé cet impact favorable de la restriction sur la santé et sur l'efficacité alimentaire, que ce soit lors d'études à grande échelle ou d'essais reproduisant des infections expérimentales. En conséquence, les stratégies de limitation de l'ingéré postsevrage sont maintenant très largement pratiquées par près de $90 \%$ des éleveurs professionnels (Lebas 2007), en paral- lèle du développement des équipements d'alimentation automatique.

Durant les dix dernières années, diverses stratégies de limitation temporaire de l'ingestion ont été étudiées chez le lapin sevré, en termes de durée de la période de restriction (1 à 5 semaines), d'intensité de la restriction (90 à $40 \%$ de l'ingéré spontané) ou de méthodes (restriction quantitative, restriction d'abreuvement, accès limité à la mangeoire...). Cet article vise donc à résumer les différents effets d'une ingestion limitée sur la santé, la croissance et l'efficacité alimentaire, la qualité de la carcasse, quelques paramètres des fonctions digestives ainsi que sur le comportement alimentaire et le bien-être du lapin.

\section{1 / Les stratégies d'ingestion limitée en cuniculture}

En élevage cunicole, les animaux ont habituellement un libre accès à l'aliment granulé (i.e. Ad Libitum = AL) et à l'eau (pipettes automatiques). Diverses techniques ont été étudiées pour contrôler l'ingestion d'aliment (ou d'eau), avec deux objectifs principaux : la maîtrise

\footnotetext{
${ }^{1}$ Cet article est adapté d'un article des mêmes auteurs publié en langue anglaise dans la revue Animal (Gidenne et al 2012b).
} 
de la qualité de la carcasse et de la viande (Perrier et Ouhayoun 1996) ; l'amélioration de l'efficacité alimentaire. Deux stratégies de restriction ont été principalement utilisées : une limitation quantitative de l'ingestion ou une restriction qualitative. Pour cette dernière, l'apport en nutriments est modifié via la composition chimique de l'aliment. Par exemple, l'apport énergétique pour les jeunes femelles reproductrices peut être restreint en utilisant des aliments riches en fibres (Rebollar et al 2011). Dans cette synthèse, nous allons traiter essentiellement des stratégies de restriction quantitative de l'ingéré, sans changement de la composition de l'aliment.

En pratique, une restriction quantitative peut être appliquée de trois façons : $i$ ) par une réduction de la durée d'accès à la mangeoire, ii) par un accès limité à l'abreuvoir et iii) en diminuant la quantité d'aliments distribués. Sachant que le lapin effectue 30 à 40 repas par jour (Prud'hon et al 1975), il y a peu de compétition pour l'accès à la mangeoire dans une cage collective. En revanche, il est difficile de limiter la consommation simplement en réduisant la largeur de la mangeoire. Ces techniques peuvent être combinées avec la durée et l'intensité de la restriction, par exemple : restriction pendant 3 semaines après le sevrage puis une période AL (ou vice versa) ou encore restriction des futures reproductrices avant le premier accouplement.

Les premières études portant sur la restriction alimentaire consistaient à restreindre la durée d'abreuvement (Lebas et Delaveau 1975). Sachant que l'ingestion de granulés est directement liée à la consommation d'eau, la réduction du temps d'abreuvement est donc une technique simple et peu coûteuse à mettre en œuvre. Ainsi, la consommation d'aliment est réduite de $18 \%$ lorsque l'abreuvement est réduit à $2 \mathrm{~h} / \mathrm{jour}$ (Boisot et al 2004), de $22 \%$ pour $1 \mathrm{~h} 30$ (Verdelhan et al 2004) et de $23 \%$ pour $1 \mathrm{~h}$ (Boisot et al 2005). Mais une forte restriction hydrique est discutable en termes de bien-être animal, en particulier dans des conditions chaudes (Foubert et al 2007, Ben Rayana et al 2008). En outre, une réduction de l'ingéré sec obtenue par un moindre abreuvement conduirait à des effets moins homogènes sur les performances et la santé, en comparaison d'une réduction quantitative de la quantité d'aliment distribuée.

Réduire la durée d'accès à la mangeoire, chaque jour ou sur une semaine, est un autre moyen de limiter la consommation. Ainsi, il est possible de réduire l'ingéré de $20 \%$, soit $80 \%$ de $\mathrm{AL}$, en maintenant un accès libre à la mangeoire (AL) pendant 5 jours suivi d'un jeûne de
2 jours (Lebas et Laplace 1982). Ce niveau de $80 \%$ de l'ingestion libre est également obtenu avec un accès à la mangeoire limité à $8 \mathrm{~h}$ par jour (Jérôme et al 1998, Szendrö et al 1988). Selon Jérôme et al (1998), si l'aliment est disponible seulement le jour (de 08:00 à 16:00 h) l'ingestion est réduite de $20 \%$ (et la croissance de $12 \%$ ) et si l'alimentation est disponible la nuit (de 16:00 à 8:00 h) la consommation n'est réduite que de $10 \%$ et la croissance de $5 \%$. En parallèle, l'Indice de Consommation (IC) n'a été amélioré que si l'aliment est accessible le jour (2,67 vs 2,93 pour AL).

Pour parvenir à un contrôle correct de l'ingestion post-sevrage, la technique la plus précise est de donner chaque jour une quantité définie de granulés, soit manuellement soit en utilisant un système de Distribution Automatique d'Aliment (DAA) maintenant relativement répandu en cuniculture. Enfin, au cours de la phase de croissance, plusieurs programmes de restriction sont possibles : réduction progressive ou non de l'ingéré, réduction par palliers, restriction continue ou en alternance, etc. La stratégie de limitation de l'ingestion doit être adaptée aux objectifs de l'éleveur: amélioration de l'état sanitaire du troupeau, réduction des coûts d'alimentation ou même réduction de l'ingestion de granulés pour stimuler la consommation de fourrage (Yakubu et al 2007).

\section{2 / Limiter l'ingestion après le sevrage réduit la mortalité et la morbidité du lapin}

L'indicateur usuel pour évaluer l'impact d'une maladie en élevage est le taux de mortalité. Plus récemment, un indicateur de la morbidité a été développé en cuniculture pour évaluer plus précisément l'incidence des symptômes cliniques durant la croissance (Gidenne et al 2010). Il peut être combiné avec la mortalité pour obtenir un index de risque sanitaire (IRS, \% = morbidité \% + mortalité \%). Un grand nombre d'animaux est nécessaire pour détecter une différence significative de mortalité entre deux traitements. Ainsi, pour détecter une différence de $10 \%$ entre deux taux de mortalité avec un risque d'erreur inférieur à 5\%, plus de 90 lapins sont nécessaires dans chaque groupe ; plus de 300 animaux par groupe sont nécessaires pour détecter une différence de $5 \%$. L'évaluation de la morbidité à l'aide de symptômes cliniques (diarrhée, parésie caecale...) est relativement simple. Cependant, dans le cas où seule une baisse de croissance est détectable, un seuil doit être défini pour classer l'animal comme morbide (tel que la moyenne moins 3 fois l'écart-type), ce qui nécessite un grand nombre d'animaux par groupe pour définir la moyenne et sa plage de variation. Par conséquent, sauf pour des expérimentations particulières comme celles impliquant l'inoculation d'un agent pathogène, nous ne retiendrons dans notre analyse de la littérature que les études utilisant un nombre élevé d'animaux permettant une estimation correcte de la mortalité et de la morbidité entre le sevrage et l'âge d'abattage.

La première étude à grande échelle portant sur les effets d'une stratégie de limitation de l'ingestion post-sevrage sur la santé du lapin a été réalisée en 2002 par le réseau GEC. Les premiers résultats (Gidenne et al 2003), indiquent que le taux de mortalité passe de 12 à $3 \%$ quand l'ingestion est limitée à $60 \%$ de l'AL pendant les trois semaines qui suivent le sevrage (Gidenne et al 2009,

Figure 1. Mortalité lors d'une infection expérimentale d'Entéropathie Epizootique du Lapin (EEL) en fonction de la stratégie de restriction alimentaire (Boisot et al 2003).

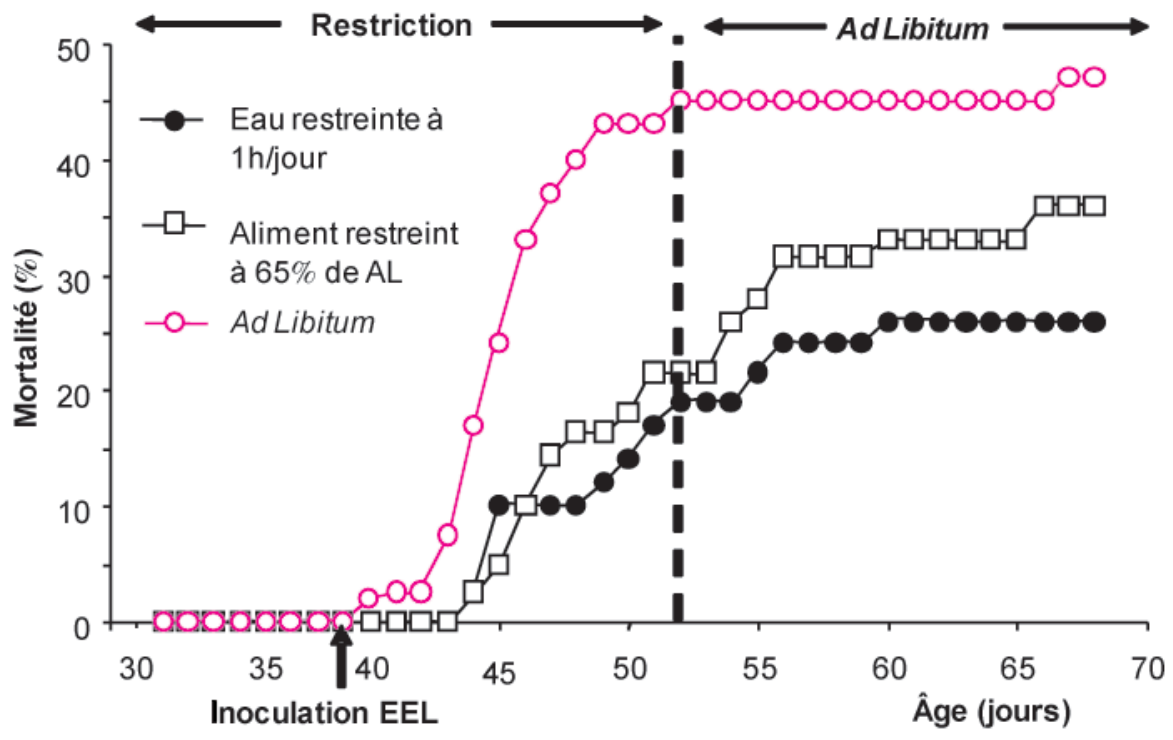


Tableau 1. Une stratégie de restriction alimentaire post-sevrage réduit la mortalité et la morbidité par troubles digestifs chez le lapin en croissance.

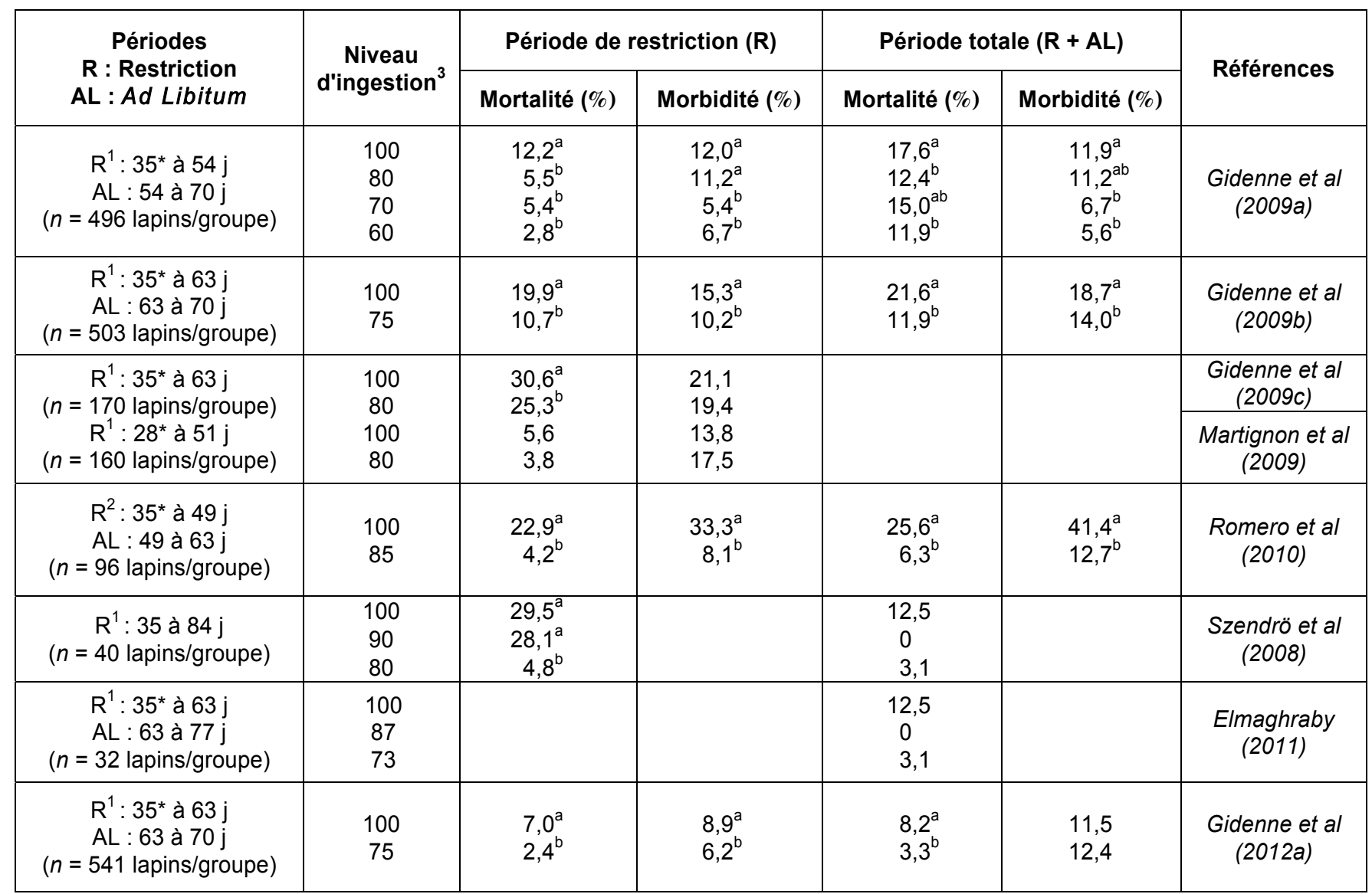

${ }_{1}^{1}$ limitation de l'ingéré par distribution quotidienne d'une quantité fixe d'aliment granulé.

2 limitation de l'ingéré par réduction du temps d'accès à la mangeoire.

${ }^{3}$ en pourcentage de l'ingestion Ad Libitum $(100 \%=A L)$.

* âge au sevrage.

$a, b$ écart significatif $(P<0,05)$ entre $A L$ et $R$, pour une même étude.

tableau 1) et que le taux de morbidité passe de 12 à $7 \%$. Des essais supplémentaires ont été effectués pour évaluer si une stratégie de restriction était efficace contre l'EEL (Boisot et al 2003) : une ingestion limitée à $65 \%$ réduit le taux de mortalité après une inoculation d'EEL (figure 1). Cet effet favorable a été confirmé par Foubert et al (2008b). En revanche, Martignon et al (2011b) indiquent qu'une ingestion limitée à $80 \%$ n'a pas d'effet significatif sur la morbidité de lapins infectés avec un colibacille modéremment entéropathogène $(0128$ : C6), ni d'effet favorable lors d'une colibacillose spontanée (Martignon et al 2009). Cet effet différent de la restriction pour ces deux pathologies digestives mérite des études complémentaires afin d'identifier les mécanismes physiologiques impliqués.

Le dispositif expérimental multi-unité du GEC (plus de 400 lapins par traitement), a permis de montrer que la mortalité et la morbidité des animaux est réduite de moitié à partir de $20 \%$ de réduction d'ingestion (Gidenne et al 2009a, tableau 1). Cet effet favorable sur la santé digestive (fréquence des diarrhées) a été confirmé dans deux autres études (Gidenne et al 2009b, Martignon et al 2009), dans lesquelles l'aliment était distibué en une ou plusieurs fractions. Ainsi, les effets de la restriction proviendraient bien de la quantité d'aliment ingéréé et non pas de la technique de distribution. Par ailleurs, il semble que l'ingéré énergétique soit impliqué dans l'effet favorable sur la santé. Gidenne et al (2009c) ont comparé un aliment témoin $(9,79$ MJ $\mathrm{d}(\mathrm{ED} / \mathrm{kg}$ ) à un aliment plus énergétique (10,90 MJ d'ED/kg), afin d'évaluer s'il est possible d'obtenir un effet favorable sur la santé sans altération de la croissance. Malheureusement, les premiers résultats ont indiqué que limiter la consommation d'un aliment énergétique ne semblait pas aussi efficace pour prévenir les troubles digestifs. Szendrő et al (2008) ont obtenu des conclusions similaires en utilisant deux génotypes de lapins en croissance.
En outre, cet effet favorable d'une restriction alimentaire sur la santé n'est visible que durant la période d'ingestion limitée. Il ne perdure pas lorque les animaux passent à une ingestion $\mathrm{AL}$ (Gidenne et al 2009, Romero et al 2010). Des résultats similaires ont également été obtenus en réduisant le niveau de consommation par une limitation de la durée d'abreuvement (Boisot et al 2004, Verdelhan et al 2004, Elmaghraby 2011).

De nombreux paramètres métaboliques sont modifiés lors d'une restriction alimentaire (Van Harten et Cardoso 2010). Le statut immunitaire de lapins restreints a été sommairement décrit à partir de quelques paramètres sanguins, et Tumova et al (2007) signalent une augmentation du nombre de lymphocytes. En perspective, il reste à analyser la réponse immunitaire et inflammatoire du lapin restreint (Romero et al 2011), comme cela a été étudié chez la souris ou l'homme (Jolly 2004), pour expliquer la relation positive entre santé et ingestion restreinte. 


\section{3 / Impact d'une restriction temporaire de l'ingéré sur la croissance, l'efficacité ali- mentaire et la qualité de la carcasse du lapin}

\section{1 / Croissance et efficacité ali- mentaire du lapin en alimenta- tion restreinte}

Réduire l'ingéré ralentit la croissance (tableau 2). Une méta-analyse de la bibliographie (figure 2) montre que la réduction de croissance est généralement inférieure à la réduction de l'ingéré : une réduction d'ingestion de $20 \%$ conduit à une baisse de vitesse de croissance de seulement $15,6 \%$ en moyenne. Ainsi, à la fin de la période de restriction, le poids vif est réduit de 7 à $10 \%$ pour des niveaux de restriction de 15 à $25 \%$ (tableau 2, Boisot et al 2003, Bergaoui et al 2008, Gidenne et al 2009a, b). De plus, l'effet d'une restriction alimentaire temporaire sur le gain de poids est généralement plus sévère au début de la période de restriction, souvent juste après le sevrage, que plus tard (Gidenne et al 2009c, Martignon et al 2010). Par ailleurs, la réduction de la croissance semble plus élevée chez des lapins nourris avec un aliment énergétique (Duperray et Guyonvarch 2009 , Gidenne et al 2009c).

Le lapin sevré s'adapte rapidement aux stratégies de restriction alimentaire (cf.
§ 5) et sans forte compétition d'accès à la mangeoire, du fait de son comportement alimentaire à repas multiples (30 à 40 par jour, Gidenne et Lebas 2006). Ainsi, la variabilité intra-cage des poids vifs individuels n'est pas accrue par la stratégie de restriction (Tudela et Lebas 2006).

Durant la restriction, l'indice de consommation est généralement légèrement réduit (de -5 à $-10 \%$, figure 2 ) malgré de larges variations entre études, probablement en lien avec la composition chimique de l'aliment. Ainsi, pour un aliment riche en énergie, Gidenne et al (2009c) ne trouvent pas d'effet de la restriction sur l'indice de consommation, tandis que pour un aliment témoin l'indice est réduit de 10\% (tableau 3 ).

Une croissance compensatrice, dont l'intensité est liée à celle de la restriction, est toujours observée lorsque les animaux sont nourris librement après une restriction (tableau 2). Pour une restriction de $40 \%$, le gain de poids est de 20 à $30 \%$ supérieur au groupe témoin toujours nourri AL et peut atteindre des valeurs très élevées, dépassant $70 \mathrm{~g} / \mathrm{j}$ (tableau 2, Gidenne et al 2009 c). Cette remarquable capacité du jeune lapin à développer une croissance compensatrice après une période de restriction avait déjà été décrite (Lebas et Laplace 1982, Tumova et al 2002, Matics et al 2008).

Toutefois, même après deux semaines d'ingestion AL, le poids vif des lapins préalablement restreints reste 5 à $10 \%$ inférieur à celui des témoins (tableau 2). Mais, il faut souligner que l'ingestion des lapins préalablement restreints reste proche de celle des témoins. Ainsi, après une période de restriction et contrairement à ce qui était attendu, il n'est pas observé de comportement de boulimie chez le jeune lapin. Ceci pourrait s'expliquer par la faible capacité stomacale à stocker de l'aliment, et qui conduit le lapin à pratiquer de nombreux repas quotidiens (Gidenne et Lebas 2006).

L'indice de consommation est donc nettement amélioré pour ces animaux à ingestion restreinte (tableau 3 ), quelle que soit la composition de l'aliment. Par conséquent, quand une stratégie de limitation de l'ingestion est appliquée, la marge sur coût alimentaire est généralement améliorée de 2 à 10\% (Duperray et Guyonvarch 2009). Cependant, environ deux ou trois jours d'élevage supplémentaires sont nécessaires pour atteindre le poids de marché français, ce qui réduit l'avantage économique. Pour d'autres systèmes cunicoles, l'intérêt d'une stratégie de restriction reste à étudier. Ainsi, une étude espagnole récente (Romero et al 2010) rapporte une altération marquée de la croissance et de l'IC, après une restriction de deux semaines. L'intérêt économique de cette stratégie de restriction serait donc moindre en système cunicole espagnol où les animaux sont abattus à un poids plus léger qu'en France (environ $2 \mathrm{~kg}$ $v s 2,3 \mathrm{~kg})$.

Figure 2. Variations de la croissance et de l'indice de consommation chez le lapin, selon l'intensité de la restriction alimentaire et pendant la période d'ingestion restreinte.

Données des tableaux 2 et 3 , limitées à la gamme usuelle de restriction, soit entre 10 et $40 \%$ (compilation bibliographique : un point $=$ une étude).

* la réduction d'ingestion est exprimée en \% de l'ingestion à volonté (AL) d'animaux contemporains (20\% de réduction correspond à une ingestion restreinte à $80 \%$ de l'ingestion libre).

** réduction de la vitesse de croissance $(\mathrm{g} / \mathrm{j})$ pendant la période de restriction exprimée en \% de la croissance d'animaux contemporains nourris librement $(A L)$.
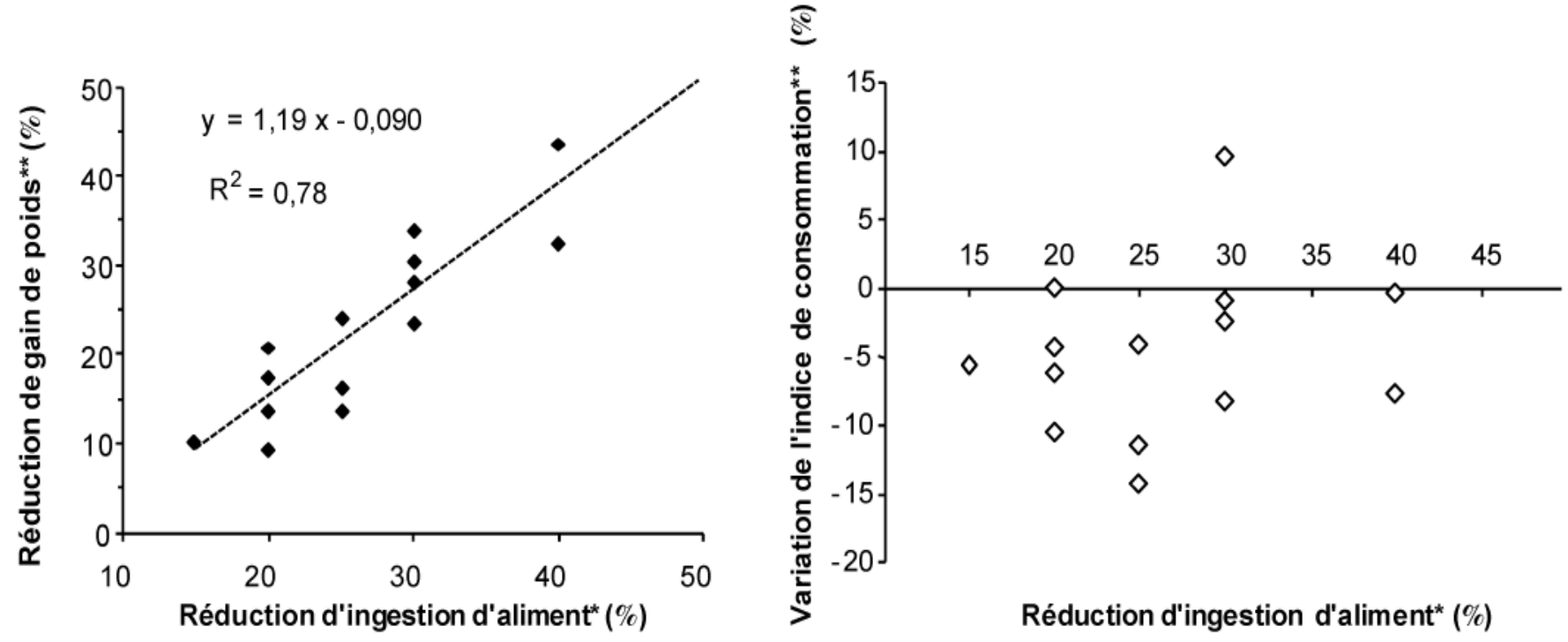

Réduction d'ingestion d'aliment* $(\%)$ 
Tableau 2. Effet de la restriction alimentaire post-sevrage sur la croissance du lapin.

\begin{tabular}{|c|c|c|c|c|c|c|c|}
\hline \multirow{2}{*}{$\begin{array}{c}\text { Périodes } \\
\mathbf{R}: \text { Restriction } \\
\text { AL : Ad Libitum }\end{array}$} & \multirow{2}{*}{$\begin{array}{c}\text { Niveau } \\
\text { d'ingestion }^{3}\end{array}$} & \multicolumn{3}{|c|}{ Gain de poids $(g / j)$} & \multicolumn{2}{|c|}{ Poids vif (g) } & \multirow{2}{*}{ Références } \\
\hline & & $\begin{array}{c}\text { pendant } \\
R\end{array}$ & $\begin{array}{l}\text { après } \\
R(A L)\end{array}$ & $\begin{array}{c}\text { total } \\
(R+A L)\end{array}$ & $\begin{array}{l}\text { en fin } \\
\text { de } R\end{array}$ & $\begin{array}{l}\text { final, en } \\
\text { fin de AL }\end{array}$ & \\
\hline \multirow{2}{*}{$\mathrm{R}^{1}: 35$ à $56 \mathrm{j}$} & 100 & $38,1^{a}$ & & & $2028^{a}$ & & \multirow{2}{*}{$\begin{array}{c}\text { Lebas et } \\
\text { Laplace (1982) }\end{array}$} \\
\hline & 70 & $25,2^{b}$ & & & $1768^{\mathrm{b}}$ & & \\
\hline \multirow{3}{*}{$\begin{array}{l}\mathrm{R}^{1}: 35^{\star} \text { à } 56 \mathrm{j} \\
\mathrm{AL}: 56 \text { à } 77 \mathrm{j}\end{array}$} & 100 & $49,6^{a}$ & $42,0^{a}$ & $45,8^{a}$ & $2175^{a}$ & $3059^{a}$ & \multirow{3}{*}{ Perrier (1998) } \\
\hline & 70 & $28,2^{b}$ & $54,6^{\mathrm{b}}$ & $41,6^{\mathrm{b}}$ & $1729^{b}$ & $2877^{b}$ & \\
\hline & 50 & $15,3^{c}$ & $62,4^{\mathrm{c}}$ & $39,0^{c}$ & $1460 c$ & $2772^{b}$ & \\
\hline \multirow{2}{*}{$\begin{array}{l}\mathrm{R}^{1,2}: 28^{*} \text { à } 49 \mathrm{j} \\
\mathrm{AL}: 63 \text { à } 70 \mathrm{j}\end{array}$} & 100 & $42,2^{a}$ & & $41,5^{a}$ & $1583^{a}$ & $2443^{a}$ & \multirow{2}{*}{$\begin{array}{l}\text { Jérome et al } \\
\quad(1998)\end{array}$} \\
\hline & 80 & $38,3^{b}$ & & $37,4^{\mathrm{b}}$ & $1505^{b}$ & $2268^{b}$ & \\
\hline \multirow{3}{*}{$\begin{array}{l}\mathrm{R}^{1}: 34^{*} \text { à } 58 \mathrm{j} \\
\mathrm{AL}: 56 \text { à } 70 \mathrm{j}\end{array}$} & 100 & $47,8^{a}$ & $38,3^{a}$ & $43,7^{a}$ & $1907^{\mathrm{a}}$ & $2519^{a}$ & \multirow{3}{*}{$\begin{array}{l}\text { Boisot et al } \\
\quad(2003)\end{array}$} \\
\hline & 80 & $41,3^{b}$ & $42,5^{\mathrm{b}}$ & $41,8^{b}$ & $1772^{\mathrm{b}}$ & $2451^{b}$ & \\
\hline & 60 & $32,3 c$ & $47,7^{\mathrm{C}}$ & $38,7^{c}$ & $1573^{\mathrm{c}}$ & $2337^{c}$ & \\
\hline \multirow{2}{*}{$\begin{array}{l}R^{1}: 32^{*} \text { à } 53 j \\
A L: 53 \text { à } 67 j\end{array}$} & 100 & $51,4^{a}$ & $38,6^{a}$ & $46,2^{a}$ & $1908^{a}$ & $2438^{a}$ & \multirow{2}{*}{$\begin{array}{l}\text { Foubert et al } \\
(2008 a)\end{array}$} \\
\hline & 70 & $36,9^{b}$ & $46,5^{\mathrm{b}}$ & $40,3^{b}$ & $1585^{\mathrm{b}}$ & $2218^{b}$ & \\
\hline \multirow{3}{*}{$\mathrm{R}^{1}: 42$ à $77 \mathrm{j}$} & 100 & $38,5^{a}$ & & & $2115^{a}$ & & \multirow{3}{*}{$\begin{array}{l}\text { Bergaoui } \\
\text { et al (2008) }\end{array}$} \\
\hline & 85 & $34,6^{b}$ & & & $1995^{b}$ & & \\
\hline & 70 & $29,4^{c}$ & & & $1740^{c}$ & & \\
\hline \multirow{4}{*}{$\begin{array}{l}R^{1}: 35^{*} \text { à } 54 j \\
A L: 54 \text { à } 70 j\end{array}$} & 100 & $40,7^{a}$ & $46,1^{a}$ & $43,5^{a}$ & $1799^{a}$ & $2468^{a}$ & \multirow{4}{*}{$\begin{array}{l}\text { Gidenne et al } \\
\quad(2009 a)\end{array}$} \\
\hline & 80 & $32,3^{b}$ & $51,1^{\mathrm{b}}$ & $40,8^{b}$ & $1624^{\mathrm{b}}$ & $2373^{b c}$ & \\
\hline & 70 & $28,4^{c}$ & $54,6^{\mathrm{c}}$ & $40,0^{b}$ & $1540^{c}$ & $2340^{c}$ & \\
\hline & 60 & $23,0^{d}$ & $58,4^{d}$ & $38,2^{c}$ & $1431^{d}$ & $2279^{d}$ & \\
\hline \multirow{2}{*}{$\begin{array}{l}\mathrm{R}^{1}: 35^{*} \text { à } 63 \mathrm{j} \\
\mathrm{AL}: 63 \text { à } 70 \mathrm{j}\end{array}$} & 100 & $46,4^{a}$ & $39,9^{a}$ & $45,1^{a}$ & $2319^{a}$ & $2612^{a}$ & \multirow{2}{*}{$\begin{array}{l}\text { Gidenne et al } \\
(2009 b)\end{array}$} \\
\hline & 75 & $38,9^{b}$ & $47,6^{\mathrm{b}}$ & $40,6^{b}$ & $2112^{\mathrm{b}}$ & $2454^{b}$ & \\
\hline \multirow{2}{*}{$\begin{array}{l}\mathrm{R}^{1}: 35^{*} \text { à } 63 \mathrm{j} \\
\mathrm{AL}: 63 \text { à } 70 \mathrm{j}\end{array}$} & 100 & $45,7^{a}$ & $49,2^{a}$ & $46,4^{a}$ & $2352^{a}$ & $2724^{a}$ & \multirow{2}{*}{$\begin{array}{l}\text { Gidenne et al } \\
\quad(2009 \mathrm{c})\end{array}$} \\
\hline & 80 & $37,8^{b}$ & $73,9^{\mathrm{b}}$ & $44,8^{b}$ & $2100^{b}$ & $2650^{\mathrm{b}}$ & \\
\hline \multirow{2}{*}{$\mathrm{R}^{1}: 28^{*}$ à $53 \mathrm{j}$} & \multirow{2}{*}{$\begin{array}{c}100 \\
75\end{array}$} & $53,4^{a}$ & & & $1349^{a}$ & & \multirow{2}{*}{$\begin{array}{l}\text { Martignon et al } \\
\quad(2010)\end{array}$} \\
\hline & & $40,5^{\mathrm{b}}$ & & & $1118^{b}$ & & \\
\hline \multirow{2}{*}{$\begin{array}{l}\mathrm{R}^{2}: 35^{*} \text { à } 49 \mathrm{j} \\
\mathrm{AL}: 49 \text { à } 63 \mathrm{j}\end{array}$} & \multirow{2}{*}{$\begin{array}{c}100 \\
79\end{array}$} & $43,6^{a}$ & $44,8^{a}$ & $44,0^{a}$ & & $1989^{a}$ & \multirow{2}{*}{$\begin{array}{c}\text { Romero et al } \\
(2010) \\
\text { (essai1) }\end{array}$} \\
\hline & & $27,1^{\mathrm{b}}$ & $54,8^{\mathrm{b}}$ & $40,4^{b}$ & & $1888^{b}$ & \\
\hline$R^{2}: 35^{*}$ à $49 j$ & 100 & $39,7^{a}$ & $45,3^{a}$ & $43,0^{a}$ & & $1997^{a}$ & Romero et al \\
\hline$A L: 49$ à $63 j$ & 85 & $29,2^{\mathrm{b}}$ & $55,7^{\mathrm{b}}$ & $40,1^{b}$ & & $1829^{b}$ & (essai 2) \\
\hline $\mathrm{R}^{1}: 35^{*}$ à $63 \mathrm{j}$ & 100 & $50,1^{a}$ & $37,4^{a}$ & $47,8^{a}$ & $2449^{a}$ & $2701^{a}$ & Gidenne et al \\
\hline$A L: 63$ à $70 j$ & 75 & $43,5^{b}$ & $44,0^{b}$ & $43,4^{b}$ & $2268^{b}$ & $2554^{b}$ & (2012a) \\
\hline
\end{tabular}

1 limitation de l'ingéré par distribution quotidienne d'une quantité fixe d'aliment granulé.

${ }^{2}$ limitation de l'ingéré par réduction du temps d'accès à la mangeoire.

${ }^{3}$ en pourcentage de l'ingestion Ad Libitum $(100 \%=\mathrm{AL})$.

* âge au sevrage.

a, b,c,d écart significatif $(P<0,05)$ entre $A L$ et $R$, pour une même étude.

3.2 / Effets d'une restriction alimentaire sur le développement des organes digestifs, le rendement en carcasse et la qualité de la viande, chez le lapin

En parallèle à la réduction de la croissance, une stratégie de restriction modifie également la composition corporelle. Selon la règle générale d'allométrie de formation des tissus chez le lapin (Cantier et al 1969, Dalle Zotte et Ouhayoun 1998), la restriction conduit à des changements différentiels de croissance entre les organes internes (tube digestif, foie....) et les tissus (mus- cles, tissus adipeux...). Les tissus ayant un développement précoce comme l'os et le tube digestif devraient être plus pénalisés par la restriction que ceux ayant un développement plus tardif comme les muscles et le tissu adipeux (Palsson 1955).

Le développement du tube digestif estimé par le poids des organes est altéré au cours de la restriction (Schlolaut et al 1978, Perrier et Ouhayoun 1996). Selon la technique de restriction, des effets différents sur le développement des organes digestifs peuvent être observés. Ainsi, le poids du foie est plus élevé chez les lapins soumis à une restriction intermittente (5 jours sur 7) comparée à une restriction quotidienne. De même, pour une même intensité de restriction (70\% de l'AL), le développement du tube digestif varie selon la technique utilisée : il est plus lourd de $12 \%$ à un âge de 67 jours pour un plan d'alimentation de 5 jours sur 7 et de $28 \%$ si la restriction est quotidienne (Lebas et Laplace 1982, Jérome et al 1998).

Mais la croissance des organes digestifs est très rapide pendant la période de croissance compensatrice associée au retour à l'ingestion AL (Ledin 1984a). Ainsi, l'estomac et d'autres organes diges- 
Tableau 3. Une stratégie de restriction alimentaire post-sevrage améliore l'efficacité alimentaire chez le lapin.

\begin{tabular}{|c|c|c|c|c|c|}
\hline \multirow{2}{*}{$\begin{array}{c}\text { Périodes } \\
\mathrm{R}: \text { Restriction } \\
\mathrm{AL}: \text { Ad } \\
\text { Libitum }\end{array}$} & \multirow[b]{2}{*}{$\begin{array}{c}\text { Niveau } \\
\text { d'ingestion }^{3}\end{array}$} & \multicolumn{3}{|c|}{ Indice de consommation } & \multirow[b]{2}{*}{ Références } \\
\hline & & $\begin{array}{c}\text { pendant } \\
R\end{array}$ & $\begin{array}{c}\text { pendant } \\
A L\end{array}$ & $\begin{array}{c}\text { période } \\
\text { totale } \\
(R+A L)\end{array}$ & \\
\hline$R^{2}: 35$ à $56 j$ & $\begin{array}{c}100 \\
70\end{array}$ & $\begin{array}{l}3,75^{\mathrm{a}} \\
4,11^{\mathrm{b}}\end{array}$ & & & $\begin{array}{l}\text { Lebas et Laplace } \\
\text { (1982) }\end{array}$ \\
\hline $\begin{array}{l}R^{1}: 35^{*} \text { à } 56 j \\
A L: 56 \text { à } 77 j\end{array}$ & $\begin{array}{c}100 \\
70 \\
50 \\
\end{array}$ & & & $\begin{array}{l}3,74^{\mathrm{a}} \\
3,66^{\mathrm{ab}} \\
3,56^{\mathrm{b}}\end{array}$ & Perrier (1998) \\
\hline $\begin{array}{l}\mathrm{R}^{1,2}: 28^{*} \text { à } 49 \mathrm{j} \\
A L: 63 \text { à } 70 \mathrm{j}\end{array}$ & $\begin{array}{l}100 \\
80\end{array}$ & $\begin{array}{l}2,19^{\mathrm{a}} \\
1,96^{\mathrm{b}}\end{array}$ & & $2,93^{\mathrm{a}}$ & $\begin{array}{l}\text { Jérome et al } \\
\text { (1998) }\end{array}$ \\
\hline $\begin{array}{l}R^{1}: 34^{*} \text { à } 58 \mathrm{j} \\
A L: 56 \text { à } 70 \mathrm{j}\end{array}$ & $\begin{array}{c}100 \\
80 \\
60\end{array}$ & $\begin{array}{l}2,36^{\mathrm{a}} \\
2,26^{\mathrm{ab}} \\
2,18^{\mathrm{b}}\end{array}$ & $\begin{array}{l}4,37^{\mathrm{a}} \\
3,21^{\mathrm{b}} \\
2,85^{\mathrm{c}}\end{array}$ & $\begin{array}{l}3,13^{\mathrm{a}} \\
2,70^{\mathrm{b}} \\
2,57^{\mathrm{b}}\end{array}$ & Boisot et al (2003) \\
\hline $\begin{array}{l}R^{1}: 32^{*} \text { à } 53 j \\
A L: 53 \text { à } 67 j\end{array}$ & $\begin{array}{c}100 \\
70\end{array}$ & $\begin{array}{l}2,19 \\
2,17\end{array}$ & $\begin{array}{l}4,04^{a} \\
2,89^{b}\end{array}$ & $\begin{array}{l}2,75^{a} \\
2,49^{b}\end{array}$ & $\begin{array}{l}\text { Foubert et al } \\
(2008 a)\end{array}$ \\
\hline$R^{1}: 42$ à $77 j$ & $\begin{array}{l}100 \\
85 \\
70 \\
\end{array}$ & $\begin{array}{l}3,93^{\mathrm{a}} \\
3,71^{\mathrm{ab}} \\
3,61^{\mathrm{b}}\end{array}$ & & & $\begin{array}{l}\text { Bergaoui et al } \\
\quad(2008)\end{array}$ \\
\hline $\begin{array}{l}R^{1}: 35^{*} \text { à } 54 j \\
A L: 54 \text { à } 70 j\end{array}$ & $\begin{array}{l}100 \\
80 \\
70 \\
60\end{array}$ & $\begin{array}{l}2,49 \\
2,49 \\
2,43 \\
2,48\end{array}$ & $\begin{array}{l}2,93^{\mathrm{a}} \\
2,43^{\mathrm{b}} \\
2,32^{\mathrm{bc}} \\
2,02^{\mathrm{c}}\end{array}$ & $\begin{array}{l}2,69^{\mathrm{a}} \\
2,54^{\mathrm{b}} \\
2,46^{\mathrm{bc}} \\
2,38^{\mathrm{c}}\end{array}$ & $\begin{array}{l}\text { Gidenne et al } \\
\quad(2009 a)\end{array}$ \\
\hline $\begin{array}{l}\mathrm{R}^{1}: 35^{*} \text { à } 63 \mathrm{j} \\
\mathrm{AL}: 63 \text { à } 70 \mathrm{j}\end{array}$ & $\begin{array}{c}100 \\
75\end{array}$ & $\begin{array}{l}2,99^{\mathrm{a}} \\
2,65^{\mathrm{b}}\end{array}$ & $\begin{array}{l}4,84^{\mathrm{a}} \\
4,53^{\mathrm{b}}\end{array}$ & $\begin{array}{l}3,31^{\mathrm{a}} \\
3,04^{\mathrm{b}}\end{array}$ & $\begin{array}{c}\text { Gidenne et al } \\
(2009 \mathrm{~b})\end{array}$ \\
\hline $\begin{array}{l}\mathrm{R}^{1}: 35^{*} \text { à } 63 \mathrm{j} \\
\mathrm{AL}: 63 \text { à } 70 \mathrm{j}\end{array}$ & $\begin{array}{c}100 \\
80\end{array}$ & $\begin{array}{l}2,83^{\mathrm{a}} \\
2,66^{\mathrm{b}}\end{array}$ & $\begin{array}{l}3,15^{\mathrm{a}} \\
2,30^{\mathrm{b}}\end{array}$ & $\begin{array}{l}2,85^{\mathrm{a}} \\
2,55^{\mathrm{b}}\end{array}$ & $\begin{array}{l}\text { Gidenne et al } \\
\text { (2009c) }\end{array}$ \\
\hline$R^{1}: 28^{*}$ à $53 j$ & $\begin{array}{c}100 \\
75 \\
\end{array}$ & $\begin{array}{l}2,19 \\
2,10 \\
\end{array}$ & & & $\begin{array}{c}\text { Martignon et al } \\
(2010)\end{array}$ \\
\hline $\begin{array}{l}R^{2}: 35^{*} \text { à } 49 j \\
A L: 49 \text { à } 63 j\end{array}$ & $\begin{array}{l}100 \\
79\end{array}$ & $\begin{array}{l}2,28 \\
2,92\end{array}$ & $\begin{array}{l}3,00 \\
2,48\end{array}$ & $\begin{array}{l}2,55 \\
2,56\end{array}$ & $\begin{array}{l}\text { Romero et al } \\
\text { (2010) (essai 1) }\end{array}$ \\
\hline $\begin{array}{l}R^{2}: 35^{*} \text { à } 49 j \\
A L: 49 \text { à } 63 j\end{array}$ & $\begin{array}{c}100 \\
85\end{array}$ & $\begin{array}{l}2,23 \\
3,07\end{array}$ & $\begin{array}{l}2,89 \\
2,36\end{array}$ & $\begin{array}{l}2,55 \\
2,58\end{array}$ & $\begin{array}{l}\text { Romero et al } \\
\text { (2010) (essai 2) }\end{array}$ \\
\hline $\begin{array}{l}\mathrm{R}^{1}: 35^{*} \text { à } 63 \mathrm{j} \\
\mathrm{AL}: 63 \text { à } 70 \mathrm{j}\end{array}$ & $\begin{array}{l}100 \\
75\end{array}$ & $\begin{array}{l}2,95^{\mathrm{a}} \\
2,53^{\mathrm{b}}\end{array}$ & $\begin{array}{l}4,92^{\mathrm{a}} \\
4,63^{\mathrm{b}}\end{array}$ & $\begin{array}{l}3,39^{\mathrm{a}} \\
3,08^{\mathrm{b}}\end{array}$ & $\begin{array}{l}\text { Gidenne et al } \\
\text { (2012a) }\end{array}$ \\
\hline
\end{tabular}

${ }_{2}^{1}$ limitation de l'ingéré par distribution quotidienne d'une quantité fixe d'aliment granulé.

2 limitation de l'ingéré par réduction du temps d'accès à la mangeoire.

3 en pourcentage de l'ingestion Ad Libitum $(100 \%=\mathrm{AL})$.

* âge au sevrage.

$a, b, c$ écart significatif $(P<0,05)$ entre $A L$ et $R$, pour une même étude.

tifs tels que le foie sont proportionnellement plus lourds chez l'animal restreint (Lebas et Laplace 1982). Enfin, le poids du tube digestif plein (organes + digesta) est environ $10 \%$ plus élevé (tableau 4) et contribue de manière significative à la croissance compensatrice après l'arrêt de la restriction. L'augmentation du poids des digesta semble être la principale cause de la hausse du poids de tube digestif plein. En conséquence, le rendement à l'abattage est réduit d'environ 2 unités (tableau 4) chez le lapin restreint. Cela ne semble pas être étroitement lié à la durée ou à l'intensité de restriction. En outre, une durée minimale de restriction semble nécessaire, puisque Tumova et al (2006) n'ont pas trouvé de variation de rendement en carcasse pour une restriction modérée de $30 \%$ de l'AL durant 1 ou 2 semaines suivie d'une alimentation libre de 2 semaines.

A l'inverse, lors d'une alimentation libre après restriction l'augmentation de la vitesse de croissance devrait profiter surtout aux tissus et organes à développement tardifs tels que le tissu adipeux (Ouhayoun 1998). Or, lors de l'abattage à un âge compris entre 10 et 11 semaines, le niveau d'engraissement de la carcasse reste toujours inférieur chez l'animal restreint. Ainsi, le poids de gras périrénal et interscapulaire est réduit d'environ $0,5 \%$. Il est corrélé négativement avec le niveau de consommation (tableau 4). De même, Gondret et al (2000) ont montré que des changements dans l'état nutritionnel à travers une restriction d'alimentation contrôlent le dépôt de lipides intramusculaires, sans changer les caractéristiques des fibres musculaires. Les autres caractéristiques de la carcasse ou de la qualité de la viande de lapin, tels que la conformation, la couleur de la viande, les pertes à la cuisson, le ratio viande/os, la capacité de rétention d'eau, le $\mathrm{pH}$ ultime, ou le type de fibre musculaire (Perrier 1998, Dalle Zotte et al 2005, Tumova et 
Tableau 4. Impacts d'une restriction temporaire de l'ingéré sur le rendement à l'abattage et quelques paramètres de la carcasse du lapin.

\begin{tabular}{|c|c|c|c|c|c|}
\hline $\begin{array}{c}\text { Périodes } \\
\text { R : Restriction } \\
\text { AL : Ad } \\
\text { Libitum }\end{array}$ & $\begin{array}{c}\text { Niveau } \\
\text { d'ingestion }\end{array}$ & $\begin{array}{l}\text { Tube digestif } \\
\text { plein (\% PV) }\end{array}$ & $\begin{array}{l}\text { Rendement à } \\
\text { l'abattage (\%) }\end{array}$ & $\begin{array}{l}\text { Tissu adipeux, } \\
\mathrm{P}+\mathrm{IS}^{4}(\% \mathrm{PV})\end{array}$ & Références \\
\hline $\begin{array}{r}\mathrm{R}^{1,2}: 28^{*} \text { à } 49 \mathrm{j} \\
\mathrm{AL}: 63 \text { à } 70 \mathrm{j}\end{array}$ & $\begin{array}{c}100 \\
80\end{array}$ & $\begin{array}{l}17,9 \\
18,9\end{array}$ & $\begin{array}{l}58,3 \\
56,9\end{array}$ & & $\begin{array}{l}\text { Jérome et al } \\
\quad(1998)\end{array}$ \\
\hline $\begin{array}{l}\mathrm{R}^{1}: 35^{*} \text { à } 60 \mathrm{j} \\
\mathrm{AL}: 60 \text { à } 81 \mathrm{j}\end{array}$ & $\begin{array}{c}100 \\
80 \\
\end{array}$ & & $\begin{array}{l}70,2^{\mathrm{a}} \\
67,6^{\mathrm{b}} \\
\end{array}$ & $\begin{array}{l}1,7^{\mathrm{a}} \\
1,3^{\mathrm{b}}\end{array}$ & $\begin{array}{c}\text { Bovera et al } \\
(2008)\end{array}$ \\
\hline $\begin{array}{l}R^{1}: 32^{*} \text { à } 53 j \\
A L: 53 \text { à } 67 j\end{array}$ & $\begin{array}{l}100 \\
70\end{array}$ & & $\begin{array}{l}57,5 \\
56,6\end{array}$ & $\begin{array}{l}2,3^{\mathrm{a}} \\
1,6^{\mathrm{b}}\end{array}$ & $\begin{array}{c}\text { Foubert et al } \\
(2008 a)\end{array}$ \\
\hline$R^{1}: 42$ à $77 j$ & $\begin{array}{c}100 \\
85 \\
70\end{array}$ & $\begin{array}{l}20,1^{\mathrm{a}} \\
21,1^{\mathrm{ab}} \\
23,4^{\mathrm{b}}\end{array}$ & $\begin{array}{l}56,4^{\mathrm{a}} \\
55,3^{\mathrm{b}} \\
54,1^{\mathrm{c}}\end{array}$ & $\begin{array}{l}1,9 \\
1,7 \\
1,3\end{array}$ & $\begin{array}{l}\text { Bergaoui et al } \\
\quad(2008)\end{array}$ \\
\hline $\begin{array}{l}R^{1}: 35^{*} \text { à } 54 j \\
A L: 54 \text { à } 70 j\end{array}$ & $\begin{array}{c}100 \\
80 \\
70 \\
60\end{array}$ & & $\begin{array}{l}56,1^{\mathrm{a}} \\
54,6^{\mathrm{b}} \\
55,4^{\mathrm{b}} \\
54,8^{\mathrm{b}}\end{array}$ & & $\begin{array}{l}\text { Gidenne et al } \\
\quad(2009 a)\end{array}$ \\
\hline $\begin{array}{l}R^{1}: 35^{*} \text { à } 63 j \\
A L: 63 \text { à } 70 j\end{array}$ & $\begin{array}{c}100 \\
75\end{array}$ & $\begin{array}{l}16,8^{a} \\
19,8^{b}\end{array}$ & $\begin{array}{l}56,4^{\mathrm{a}} \\
54,5^{\mathrm{b}}\end{array}$ & $\begin{array}{l}2,3^{\mathrm{a}} \\
1,7^{\mathrm{b}}\end{array}$ & $\begin{array}{l}\text { Gidenne et al } \\
\quad(2009 d)\end{array}$ \\
\hline $\begin{array}{l}\mathrm{R}^{1}: 35^{\star} \text { à } 63 \mathrm{j} \\
\mathrm{AL}: 63 \text { à } 70 \mathrm{j}\end{array}$ & $\begin{array}{l}100 \\
75\end{array}$ & $\begin{array}{l}16,1^{\mathrm{a}} \\
17,9^{\mathrm{b}}\end{array}$ & $\begin{array}{l}58,2^{a} \\
56,8^{b}\end{array}$ & $\begin{array}{l}1,6^{\mathrm{a}} \\
1,1^{\mathrm{b}}\end{array}$ & $\begin{array}{l}\text { Travel et al } \\
\text { (2011) }\end{array}$ \\
\hline
\end{tabular}

\footnotetext{
${ }^{1}$ limitation de l'ingéré par distribution quotidienne d'une quantité fixe d'aliment granulé.

2 limitation de l'ingéré par réduction du temps d'accès à la mangeoire.

${ }^{3}$ en pourcentage de l'ingestion Ad Llibitum $(100 \%=\mathrm{AL})$.

${ }^{4}$ tissu adipeux périrénal $(P)+$ interscapulaire (IS).

* âge au sevrage.

$a, b$ écart significatif $(P<0,05)$ entre $A L$ et $R$, pour une même étude.

$\% \mathrm{PV}=$ pourcentage du Poids Vif à l'abattage.
}

al 2006, Gidenne et al 2009a, d, Travel et al 2011) ne sont pas modifiés par une stratégie de limitation temporaire de l'ingestion.

\section{4/Effet d'une restriction alimentaire sur les fonc- tions de digestion du lapin}

\section{1 / Impacts d'une restriction sur l'efficacité de digestion et le transit}

L'amélioration de l'indice de consommation observée pendant ou après la restriction (tableau 3 ) pourrait être associée à une amélioration de la digestion des aliments. Ainsi, la plupart des études montrent une amélioration des coefficients de digestibilité fécale, soit au cours de la restriction (tableau 5), ou après restriction pendant la période d'alimentation AL (tableau 6). Toutefois, cette amélioration est peu corrélée avec l'intensité de la restriction, mais dépendrait de sa durée. Par exemple, 7 jours après l'application de la stratégie de restriction, le coefficient de digestibilité fécale de la matière organique n'a pas été modifié, même pour une réduction d'ingestion de $40 \%$ (Gidenne et Feugier 2009). De même, sans délai d'adapta- tion Diaz Arca et al (1999) n'ont pas détecté d'effet du niveau d'ingestion sur la digestion, même lorsque l'ingéré est réduit de $90 \%$. La restriction d'ingestion améliorerait la digestion après un délai d'adaptation d'au moins 8 à 10 jours.

Une augmentation de la digestion des protéines a été souvent observée chez le lapin restreint, en croissance (Xiccato et al 1992, Gidenne et Feugier 2009, Gidenne et al 2011), et chez l'adulte (Lebas 1979, Xiccato et Cinetto 1988,

Figure 3. Le transit digestif est plus lent chez le lapin en alimentation restreinte (Gidenne et Feugier 2009).

Temps de séjour moyen, oro-rectal, mesuré chez des lapins de 7 semaines, et nourris librement $(A L)$ ou dont l'ingestion est limitée à 80 ou $60 \%$ de l'ingestion libre.

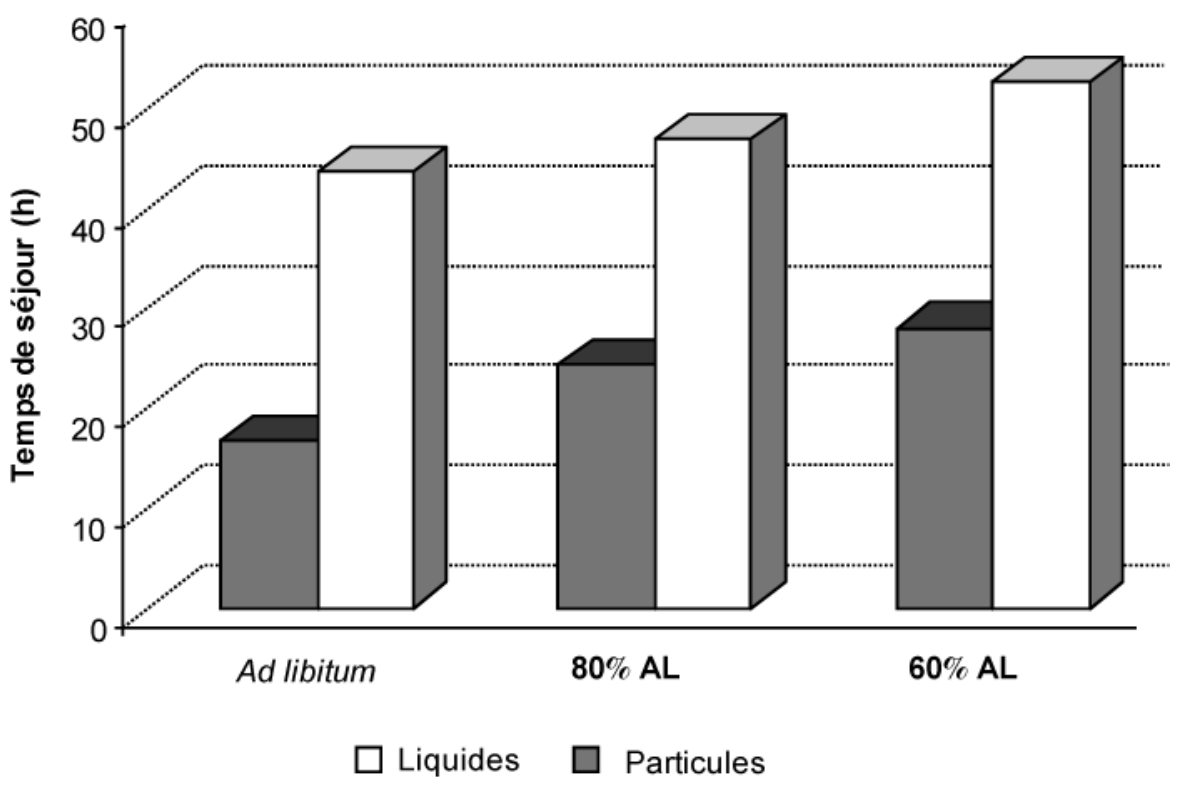


Tableau 5. Digestion du lapin soumis à une restriction alimentaire.

\begin{tabular}{|c|c|c|c|c|c|}
\hline \multirow{2}{*}{$\begin{array}{c}\text { Périodes } \\
\mathrm{R}: \text { Restriction } \\
\mathrm{AL}: \text { : Ad Libitum }\end{array}$} & \multirow[b]{2}{*}{$\begin{array}{c}\text { Niveau } \\
\text { d'ingestion }^{2}\end{array}$} & \multicolumn{3}{|c|}{ Digestibilité totale $(\%)^{3}$} & \multirow[b]{2}{*}{ Références } \\
\hline & & $\begin{array}{c}\text { Matière } \\
\text { organique }\end{array}$ & $\begin{array}{l}\text { Protéines } \\
\text { brutes }\end{array}$ & NDF & \\
\hline $\mathrm{R}^{1}: 35^{\star}$ à $72 \mathrm{j}$ & $\begin{array}{c}100 \\
50 \\
60\end{array}$ & $\begin{array}{l}66,8^{\mathrm{a}} \\
71,0^{\mathrm{c}} \\
68,2^{\mathrm{b}}\end{array}$ & $\begin{array}{l}77,9^{\mathrm{a}} \\
84,0^{\mathrm{c}} \\
82,0^{\mathrm{b}}\end{array}$ & & Ledin (1984a) \\
\hline $\mathrm{R}^{1}: \approx 45 \mathrm{à} 75 \mathrm{j}$ & $\begin{array}{c}100 \\
60\end{array}$ & $\begin{array}{l}57,5^{\mathrm{a}} \\
66,8^{\mathrm{b}}\end{array}$ & $\begin{array}{l}64,6^{\mathrm{a}} \\
74,3^{\mathrm{b}}\end{array}$ & $\begin{array}{l}27,8 \\
52,3\end{array}$ & Ledin (1984b) \\
\hline$R^{1}: 40$ à $64 j$ & $\begin{array}{c}100 \\
75\end{array}$ & $\begin{array}{l}63,7^{\mathrm{a}} \\
64,7^{\mathrm{b}}\end{array}$ & $\begin{array}{l}70,0^{\mathrm{a}} \\
72,5^{\mathrm{b}}\end{array}$ & $\begin{array}{l}19,6 \\
20,0\end{array}$ & $\begin{array}{c}\text { Xiccato et al } \\
\text { (1992) }\end{array}$ \\
\hline $\begin{array}{l}\mathrm{R}: 45 \text { à } 60 \mathrm{j} \\
\text { (digestibilité } \\
\text { calculée pour une } \\
\text { période de } 15 \mathrm{j} \text { ) }\end{array}$ & $\begin{array}{l}100 \\
60 \\
40 \\
10\end{array}$ & $\begin{array}{l}76 \\
75 \\
74 \\
73\end{array}$ & $\begin{array}{l}85 \\
87 \\
86 \\
85\end{array}$ & $\begin{array}{l}25 \\
23 \\
21\end{array}$ & $\begin{array}{l}\text { Diaz Arca et al } \\
\text { (1999) }\end{array}$ \\
\hline$R^{1}$ *35 à $85 j$ & $\begin{array}{c}100 \\
90\end{array}$ & $\begin{array}{l}63,5^{\mathrm{a}} \\
66,0^{\mathrm{b}}\end{array}$ & $\begin{array}{l}79,8 \\
79,9\end{array}$ & $\begin{array}{l}19,1^{\mathrm{a}} \\
24,5^{\mathrm{b}}\end{array}$ & $\begin{array}{l}\text { Di Méo et al. } \\
\quad(2007)^{4}\end{array}$ \\
\hline$R^{1} 42$ à $49 j$ & $\begin{array}{l}100 \\
\approx 60\end{array}$ & $\begin{array}{l}39,2 \\
44,2\end{array}$ & $\begin{array}{l}68,6^{a} \\
70,6^{b}\end{array}$ & & $\begin{array}{c}\text { Tumova et al } \\
\text { (2007) }\end{array}$ \\
\hline$R^{1}: 35^{*}$ à $54 j$ & $\begin{array}{c}100 \\
80 \\
70 \\
60 \\
\end{array}$ & $\begin{array}{l}71,9 \\
71,7 \\
72,7 \\
72,8 \\
\end{array}$ & $\begin{array}{l}82,1 \\
82,1 \\
83,5 \\
83,7 \\
\end{array}$ & $\begin{array}{l}29,9 \\
29,3 \\
30,1 \\
30,1 \\
\end{array}$ & $\begin{array}{c}\text { Gidenne et } \\
\text { Feugier (2009) }\end{array}$ \\
\hline $\begin{array}{l}\mathrm{R}^{1}: 35^{*} \text { à } 63 \mathrm{j} \\
\text { aliment témoin } \\
\mathrm{R}^{1}: 35^{\star} \text { à } 63 \mathrm{j} \\
\text { aliment riche } \\
\text { en énergie }\end{array}$ & $\begin{array}{c}100 \\
80 \\
100 \\
80\end{array}$ & $\begin{array}{l}64,2^{\mathrm{a}} \\
58,1^{\mathrm{b}} \\
63,8^{\mathrm{a}} \\
68,9^{\mathrm{b}}\end{array}$ & $\begin{array}{l}72,0 \\
71,0 \\
73,7 \\
80,8\end{array}$ & $\begin{array}{l}29,1^{\mathrm{a}} \\
17,0^{\mathrm{b}} \\
32,5^{\mathrm{a}} \\
40,9^{\mathrm{b}}\end{array}$ & $\begin{array}{l}\text { Gidenne et al } \\
\quad(2009 c)\end{array}$ \\
\hline$R^{1}: 35^{\star}$ à $63 j$ & $\begin{array}{c}100 \\
75\end{array}$ & $\begin{array}{l}60,3^{\mathrm{a}} \\
62,7^{\mathrm{b}}\end{array}$ & $\begin{array}{l}70,1^{\mathrm{a}} \\
76,2^{\mathrm{b}}\end{array}$ & $\begin{array}{l}14,5 \\
17,3\end{array}$ & $\begin{array}{c}\text { Gidenne et al } \\
(2009 d)\end{array}$ \\
\hline $\begin{array}{l}\mathrm{R}^{1}: 35^{*} \text { à } 63 \mathrm{j} \\
\text { aliment témoin } \\
\mathrm{R}^{1}: 35^{\star} \text { à } 63 \mathrm{j} \\
\text { aliment riche en } \\
\text { protéines }\end{array}$ & $\begin{array}{c}100 \\
75 \\
100 \\
75\end{array}$ & $\begin{array}{l}59,3^{\mathrm{a}} \\
64,3^{\mathrm{b}} \\
60,6^{\mathrm{a}} \\
64,1^{\mathrm{b}}\end{array}$ & $\begin{array}{l}62,6^{a} \\
71,9^{b} \\
71,0 \\
74,7\end{array}$ & $\begin{array}{l}33,1^{\mathrm{a}} \\
39,0^{\mathrm{b}} \\
29,9^{\mathrm{a}} \\
36,2^{\mathrm{b}}\end{array}$ & $\begin{array}{l}\text { Gidenne et al } \\
\quad(2011)\end{array}$ \\
\hline
\end{tabular}

\footnotetext{
${ }^{1}$ limitation de l'ingéré par distribution quotidienne d'une quantité fixe d'aliment granulé.

${ }^{2}$ en pourcentage de l'ingestion Ad Libitum $(100 \%=\mathrm{AL})$.

${ }^{3}$ digestibilité mesurée pendant la période de restriction.

${ }^{4}$ digestibilité calculée à $77 \mathrm{j}$ d'âge, avec une méthode de marquage (acid insoluble ash).

* âge au sevrage.

$a, b, c$ écart significatif $(P<0,05)$ entre $A L$ et $R$, pour une même étude.

Protéines brutes $=\mathrm{N} \times 6,25 ;$ NDF $=$ Neutral Detergent Fibre.
}

Fodor et al 2001). Ces auteurs ont constaté une meilleure digestibilité de l'énergie chez les animaux restreints, mais peu d'améliorations de la digestion des lipides et des fibres.

L'effet d'une restriction semble être moindre chez des lapins adultes (Ledin 1984b), bien que pour des aliments riches en fibres Gidenne (1987) ne trouve pas d'effet de la restriction sur la digestion. Par ailleurs, Lebas (1979) observe une digestibilité plus élevée chez la femelle gravide restreinte.

La bibliographie n'indiquant pas une relation cohérente entre la digestion et la restriction alimentaire, on peut donc supposer que la composition chimique de l'aliment joue un rôle clé. Ainsi, une interaction significative a été montrée entre le niveau d'ingestion et la concentration énergétique de l'aliment sur la digestion de l'énergie (tableau 5, Gidenne et al 2009c). Il en est de même en ce qui concerne la digestion des protéines d'un aliment riche en protéines et rationné (tableau 5, Gidenne et al 2011). Ces phénomènes d'interactions restent actuellement inexpliqués.

En revanche, l'amélioration de l'efficacité digestive chez l'animal restreint pourrait s'expliquer par des changements physiologiques intestinaux (sécrétion d'enzymes, potentiel d'absorption de la muqueuse...) et de temps de transit des digesta. Ainsi, le temps de rétention des particules dans le segment caecocolique du lapin est accru de $65 \%$ lorsque l'ingestion est réduite de $40 \%$ (figure 3). Des résultats similaires ont été observés par Ledin (1984b), tandis qu'avec des régimes fibreux, Gidenne et al (1987) mesurent une augmentation de $25 \%$ du temps de séjour moyen oro-rectal chez des lapins adultes. Par ailleurs, Fioramonti et Ruckebush (1974) indiquent que si le lapin adulte est nourri avec un seul repas par jour (et donc restreint), les contractions du caecum sont plus nombreuses avant le repas. En outre, un animal affamé ingère une grande quantité de nourriture dans un court laps de temps, ce qui prolonge l'activité motrice de l'intestin grêle (Ruckebusch et al 1971). Par conséquent, l'application d'une 
Tableau 6. Digestion du lapin nourri à volonté après une période d'ingestion restreinte.

\begin{tabular}{|c|c|c|c|c|}
\hline \multirow{2}{*}{$\begin{array}{c}\text { Périodes } \\
\mathrm{R}: \text { Restriction } \\
\mathrm{AL}: \text { Ad Libitum }\end{array}$} & \multirow[b]{2}{*}{$\begin{array}{c}\text { Niveau } \\
\text { d'ingestion }^{2}\end{array}$} & \multicolumn{2}{|c|}{ Digestibilité totale $(\%)^{3}$} & \multirow[b]{2}{*}{ Références } \\
\hline & & $\begin{array}{c}\text { Matière } \\
\text { organique }\end{array}$ & $\begin{array}{l}\text { Protéines brutes } \\
(\mathrm{N} \times 6,25)\end{array}$ & \\
\hline $\begin{array}{l}R^{1}: 35 \text { à }-72 j \\
A L: 72 \text { à } 99 j\end{array}$ & $\begin{array}{c}100 \\
50 \\
60\end{array}$ & $\begin{array}{l}64,4^{\mathrm{ab}} \\
62,9^{\mathrm{a}} \\
66,7^{\mathrm{b}}\end{array}$ & $\begin{array}{l}72,4^{\mathrm{a}} \\
73,6^{\mathrm{ab}} \\
74,2^{\mathrm{b}}\end{array}$ & Ledin (1984a) \\
\hline $\begin{array}{l}R^{1}: 42 \text { à } 56 j \\
A L: 56 \text { à } 63 j\end{array}$ & $\begin{array}{l}100 \\
\approx 60\end{array}$ & & $\begin{array}{l}77,2 \\
78,7\end{array}$ & Tumova et al (2007) \\
\hline $\begin{array}{l}\mathrm{R}^{1}: 35^{*} \text { à } 63 \mathrm{j} \\
\text { aliment témoin } \\
\mathrm{R}^{1}: 35^{*} \text { à } 63 \mathrm{j} \\
\text { aliment riche } \\
\text { en protéines }\end{array}$ & $\begin{array}{c}100 \\
75 \\
100 \\
75\end{array}$ & $\begin{array}{l}57,3 \\
56,9 \\
58,5 \\
57,8\end{array}$ & $\begin{array}{l}61,4 \\
58,6 \\
67,0 \\
65,8\end{array}$ & Gidenne et al (2011) \\
\hline
\end{tabular}

\footnotetext{
2 en pourcentage de l'ingestion Ad Libitum (100\% = AL).

${ }^{3}$ digestibilité mesurée après une période d'ingestion restreinte, lorsque les lapins sont ensuite nourris librement et en croissance compensatrice.

* âge au sevrage.

$a, b$ écart significatif $(P<0,05)$ entre $A L$ et $R$, pour une même étude.
}

stratégie de restriction chez le lapin modifie fortement l'activité motrice dans tous les segments du tube digestif, et conduit à une importante période de vacuité digestive entre les repas.

\section{2 / Restriction alimentaire et physiologie digestive}

L'application d'une stratégie de restriction pourrait nuire à la maturation de l'intestin qui évolue rapidement chez le jeune lapin. Par exemple, la hauteur des villosités iléales, la surface et la profondeur des cryptes augmentent après le sevrage (Gallois et al 2005). Elles ne sont pas affectées à 53 jours d'âge par une réduction de $25 \%$ de l'ingestion appliquée depuis le sevrage à 28 jours (Martignon et al 2010). En outre, la sécrétion d'enzymes digestives est liée à la disponibilité en substrat et donc au niveau de la consommation. Cependant en période de restriction, l'activité de la maltase dans l'iléon n'est pas modifiée (Martignon et al 2010).

Sachant que la ration est totalement consommée en 6 à $10 \mathrm{~h}$ pour des lapins restreints à $80 \%$ ( $v s$ en $24 \mathrm{~h}$ pour une alimentation AL), le flux de digesta dans l'estomac est donc très élevé juste après la distribution de l'aliment. Ainsi, le $\mathrm{pH}$ gastrique est supérieur (dans l'antrum et le fundus) chez le lapin restreint (Gidenne et Feugier 2009, Martignon et al 2010) comparé à celui qui est nourri AL. Cette élévation du $\mathrm{pH}$ est probablement transitoire et pourrait être liée à un effet de dilution de l'acide gastrique par l'important bol alimentaire réalisé par l'animal restreint. A l'inverse, 5 à $6 \mathrm{~h}$ après une distribution d'aliment, un $\mathrm{pH}$ plus bas est observé dans le caecum et est associé à une élévation de la concentration en Acides Gras Volatils (AGV, Gidenne et Feugier 2009). De même, chez le lapin restreint recevant un repas à 08:00 h, le flux iléal est élevé vers 13:00 h et associé à un pic fermentaire dans le caecum (Gidenne et Bellier 1992). Par ailleurs, Maertens et Peeters (1988) comme Taranto et al (2003) ont observé un $\mathrm{pH}$ plus élevé et une faible concentration en AGV dans le caecum chez de jeunes lapins restreints, car les mesures avaient été faites dans la matinée ( 9 à $10 \mathrm{~h}$ ) juste après la distribution du repas et donc avant le pic de fermentation dans le caecum. Par conséquent, lorsque l'échantillonnage dans le caecum tient compte du délai depuis le pic d'ingestion (Martignon et al 2010), la restriction ne modifie pas les paramètres physicochimiques ( $\mathrm{pH}, \mathrm{AGV})$ du biotope caecal, bien que le potentiel d'oxydo-réduction soit réduit de $10 \%$. L'activité fibrolytique des bactéries caecales n'est pas modifiée par le niveau de consommation (Gidenne et Feugier 2009, Martignon et al 2010). De même, le niveau de consommation n'a pas d'effet majeur sur la concentration caecale en bactéries ni sur la structure des communautés bactériennes ou leur diversité (Martignon et al 2010) estimée par empreinte moléculaire. Cette absence d'effet peut provenir du fait que la composition du flux iléal est relativement constante, malgré la réduction du niveau d'ingestion, et que le caecum possède un pouvoir tampon assez élevé. Cependant, la diversité bactérienne et la structure du microbiote, estimées par analyse d'empreintes moléculaires, ne prend en compte que les populations bactériennes dominantes.

\section{5 / Conséquences d'une res- triction temporaire sur le comportement alimentaire et le bien-être du lapin}

Le lapin nourri AL présente une ingestion relativement régulière sur le nycthémère (figure 4), répartie en 30 à 40 repas. Toutefois l'ingestion est un peu plus élevée (au maximum 10\% de l'ingéré quotidien) 2 à $4 \mathrm{~h}$ après la fin de la période diurne et un peu plus faible 2 à $4 \mathrm{~h}$ après la fin de la période nocturne, ce qui correspond à la période de caecotrophie (Gidenne et Lebas 2006). La restriction modifie ce comportement puisqu'une ration correspondant à $85 \%$ de l'ingestion AL est totalement consommée dans les 8 heures suivant la distribution (figure 5, Tudela et Lebas 2006), ce qui signifie que le lapin est à jeun pendant $16 \mathrm{~h}$. Lorsque les lapins ont accès à deux mangeoires (figure 5 , restriction $2 \mathrm{~F}$ ), la compétition pour se nourrir semble plus faible puisque les lapins mangent leur ration plus lentement, en 12 heures au lieu de 8 heures (Tudela et Lebas 2006). Selon l'intensité de la restriction, la durée du jeûne va donc varier et peut dépendre d'autres facteurs tels que les conditions climatiques. Sous un climat chaud, Bergaoui et al (2008) signalent que la ration est consommée en $16 \mathrm{~h}$ pour un niveau d'ingestion de $85 \%$, et en moins de $10 \mathrm{~h}$ pour un niveau d'ingestion de $70 \%$.

Comme le montre la figure 4, le lapin s'adapte très rapidement à une stratégie de restriction. Des lapins restreints depuis 8 jours ingèrent $40 \%$ de leur ration 
Figure 4. Profils d'ingestion du lapin nourri librement Ad Libitum ( $A L$ ) ou en ingestion limitée à 75\% de AL (Martignon et al 2009). $A, B$ : mesures réalisées 8 jours $(A)$ et 16 jours $(B)$ après l'application de la restriction depuis le sevrage $(28 \mathrm{j})$

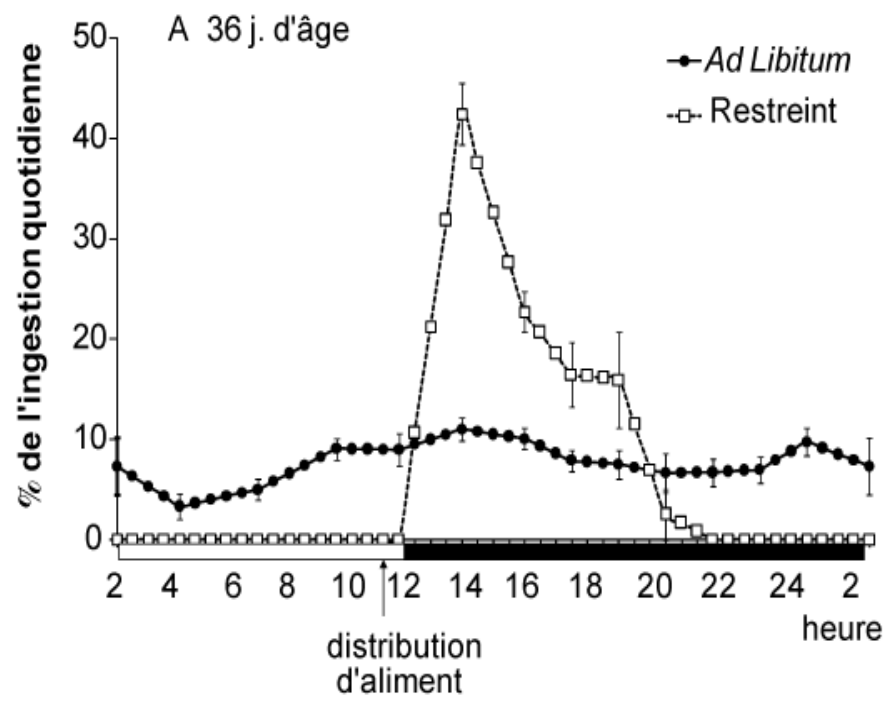

d'aliment

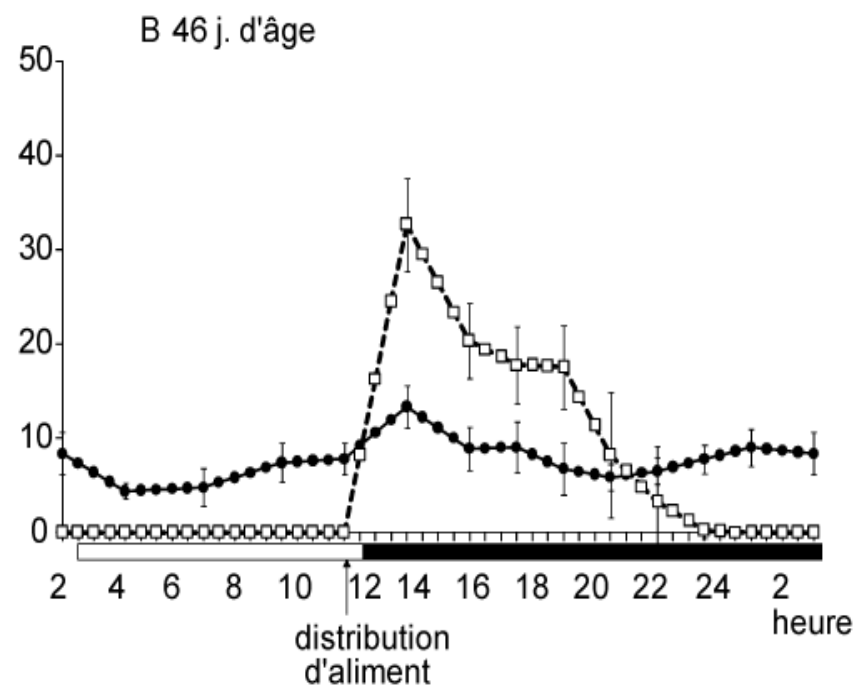

période diurne quotidienne en seulement $2 \mathrm{~h}$ (Martignon et al 2009). L'activité d'alimentation est donc intense après la distribution de la ration et le nombre moyen de repas passe de $41 / \mathrm{j}$ chez l'animal nourri AL à $27 / \mathrm{j}$ chez l'animal restreint à $80 \%$ (Martignon et al 2011a), alors que le temps moyen d'ingestion passe de $3 \mathrm{~h} 17 \mathrm{~min}$ à $2 \mathrm{~h} 30 \mathrm{~min}$. En parallèle, le nombre d'abreuvements est également réduit (de 47 à 38 par jour) chez le lapin restreint, bien que la quantité d'eau consommée soit plus élevée pour les lapins soumis à une restriction. Ainsi, Boisot et al (2005) observent que le ratio eau/aliment est doublé pour une ingestion limitée à $65 \%(3,46 v s 1,68$; figure 6). Ces auteurs ont aussi rapporté qu'une réduction de $35 \%$ de l'ingestion peut être atteinte si l'accès à l'eau est restreint à une heure par jour, mais dans ce cas le rapport eau/aliment chute à 1,20 . Une telle stratégie de restriction via l'abreuvement est peu encouragée en raison des conséquences sur le bien-être.

Lors du retour à l'alimentation $\mathrm{AL}$, le nombre de repas, leur durée et le nombre d'abreuvements deviennent plus élevés que celui des animaux témoins (48vs 38 repas par jour; $4 \mathrm{~h} 17$ vs $2 \mathrm{~h} 32 \mathrm{~min}$ pour la durée des repas ; 40 vs 36 par jour pour le nombre d'abreuvements) (Martignon et al 2011a). Toutefois le lapin s'adapte rapidement lors du retour à l'alimentation libre : son comportement alimentaire devient similaire au témoin dans un délai de 2 à 3 jours. En réponse à ces modifications rapides de comportement alimentaire, le profil d'excrétion des fèces et la caecotrophie sont fortement modifiés (figure 7). Lors d'une restriction, le pic d'excrétion fécale a lieu entre 5 et $8 \mathrm{~h}$ après la distribution de la ration, soit environ 3 à $4 \mathrm{~h}$ après le pic d'ingestion. La période de caecotrophie est alors placée environ 8 à $10 \mathrm{~h}$ après la distribution de l'aliment, comme cela avait été observé par Fioramonti et Ruckebusch (1974) chez des lapins adultes nourris une fois par jour. Par ailleurs, cette modification de profil d'excrétion ne modifie pas le ratio fèces/caecotrophes (Martignon 2010).
Enfin, soulignons que durant la première heure qui suit la distribution de la ration, l'accès à la mangeoire fait l'objet d'une légère compétition, mais sans que l'on puisse détecter des comportements agressifs entre animaux et encore moins des blessures (Martignon et al 2011a).

L'application d'une stratégie temporaire de limitation de l'ingestion ne

Figure 5. Ingestion post prandiale du lapin nourri librement ( $A L)$ ou en ingestion limitée à $85 \%$ de AL (Tudela et Lebas 2006).

Ingestion mesurée sur des lapins âgés de 10 semaines.

Restriction $1 \mathrm{~F}$ : aliment distribué dans une seule mangeoire, en quantité limitée à $85 \%$ de $\mathrm{AL}$.

Restriction $2 \mathrm{~F}$ : aliment distribué dans deux mangeoires en quantité limitée à 85\% de AL.

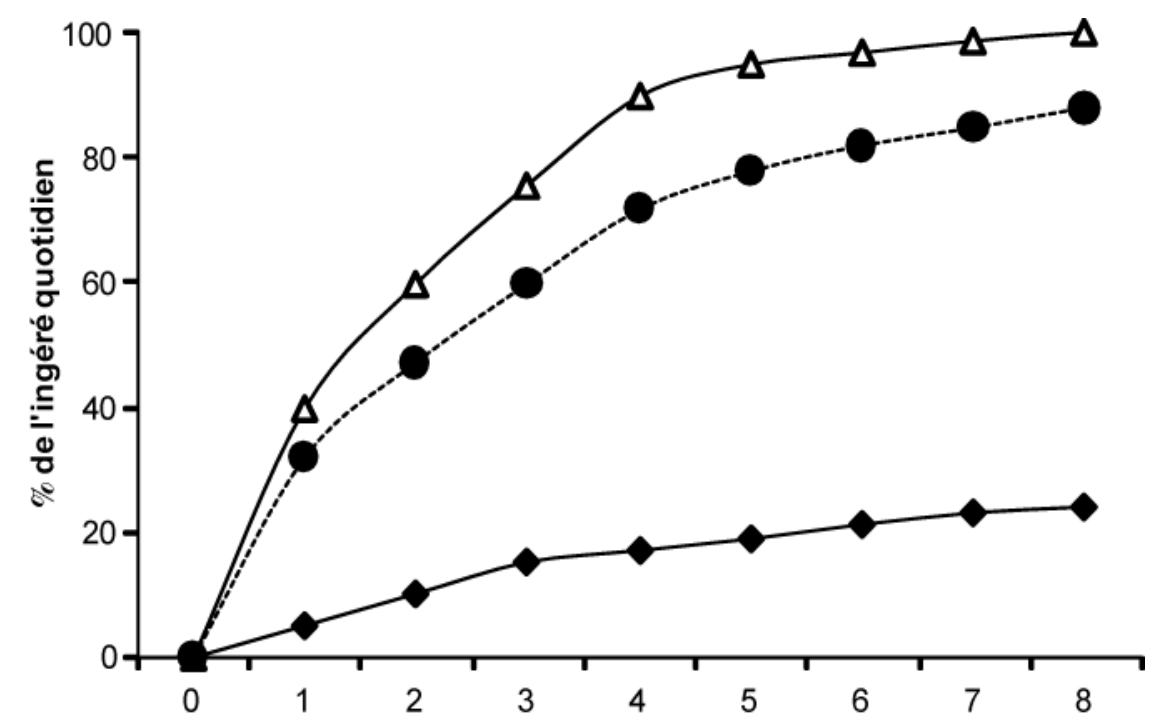

Délai après distibution de la ration $(h)$

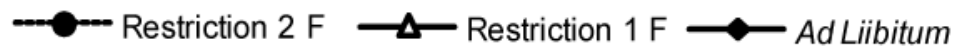


respecte pas totalement l'une des cinq règles du bien-être animal (http://www. fawc.org.uk/ freedoms.htm): l'absence de faim. Cependant, en considérant une période de $24 \mathrm{~h}$, le lapin exprime une faim transitoire (excepté le cas de la restriction de l'abreuvement) et sa croissance n'est pas fortement réduite si l'on considère des stratégies usuelles de restriction (entre 60 et $80 \%$ de l'ingestion libre pendant 2 à 4 semaines). En revanche, l'application d'une stratégie de res-

Figure 6. Le ratio eau sur aliment ingéré est fortement modifié selon la stratégie de restriction appliquée au lapin en croissance (Boisot et al 2005). Croissance mesurée entre $32 \mathrm{j}$ (sevrage) et 53 j d'âge.

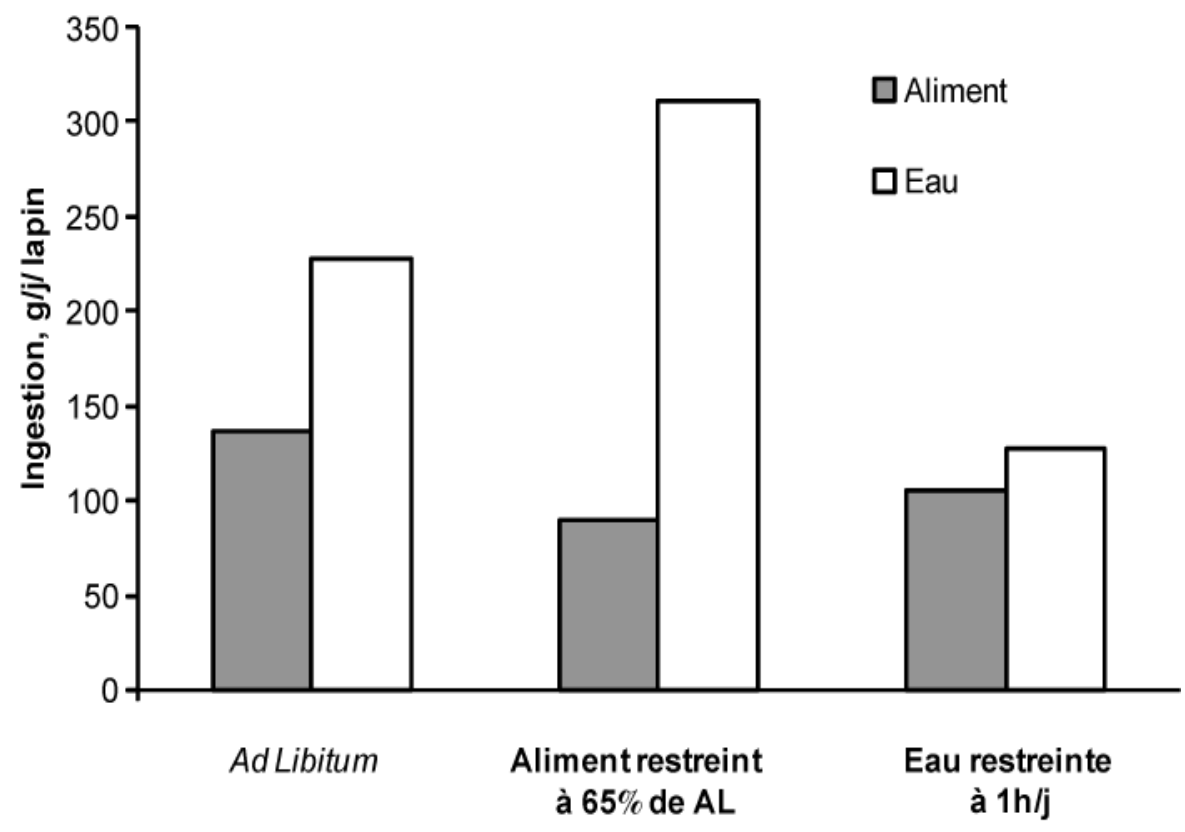

Figure 7. Profil d'excrétion fécale chez le lapin, nourri librement (AL) ou en ingestion limitée à $75 \%$ de $A L$ (Martignon et al 2009). Mesures réalisées à 47 jours d'âge, soit 19 jours après l'application de la restriction au sevrage (28 j).

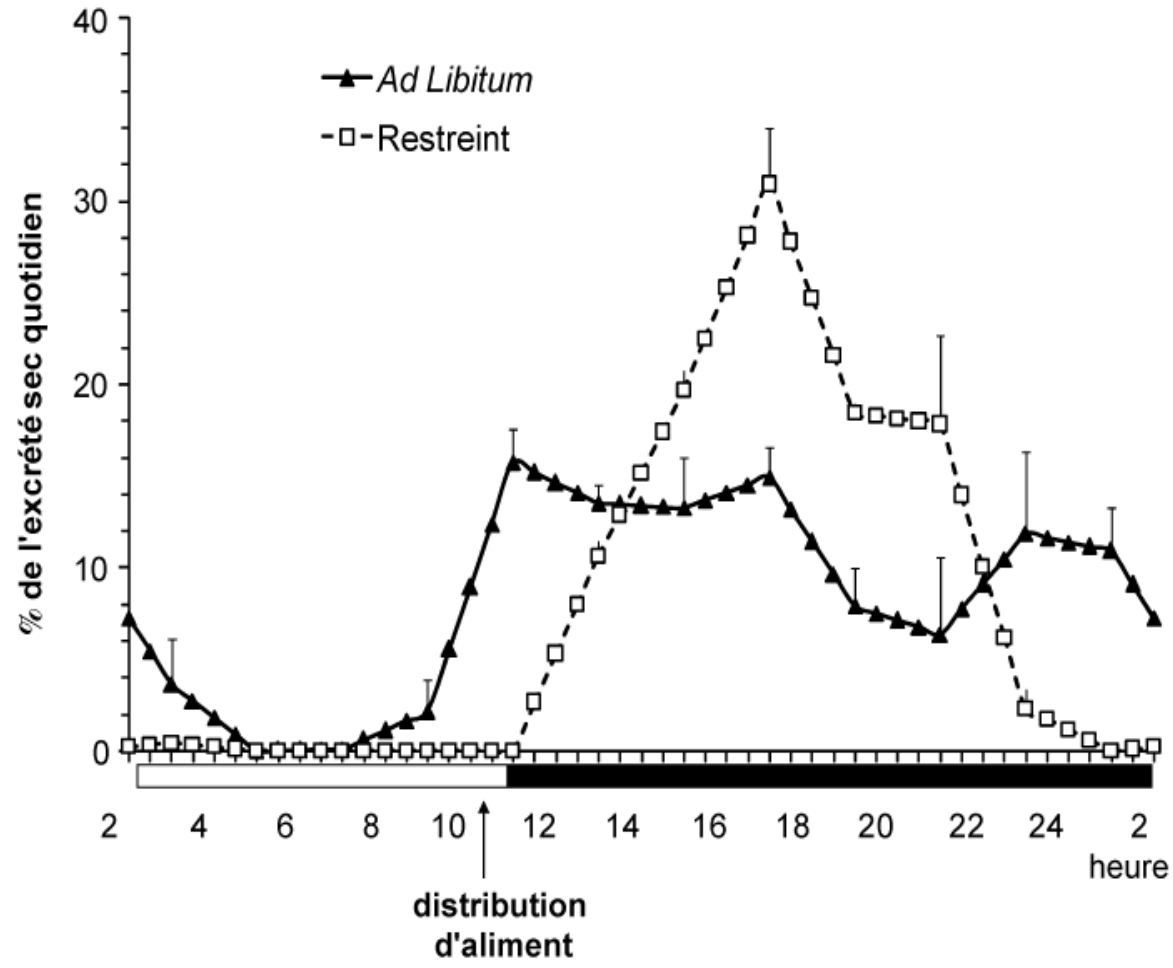

mériode nocturne $\square$ période diurne

triction permet de mieux respecter la troisième règle du bien-être animal : l'absence de douleur et de maladie, puisque de nombreuses études démontrent l'amélioration de la santé digestive du lapereau.

\section{Conclusion}

Depuis 2003, l'usage de stratégies de limitation temporaire de l'ingestion après le sevrage s'est largement répandu chez les cuniculteurs français. Ces stratégies ont été combinées à l'amélioration des équipements pour contrôler la distribution des aliments, notamment par l'utilisation de systèmes de Distribution Automatique d'Aliment (DAA) par chaînes de convoyage (Weissman et al 2009). Actuellement, environ 90\% des cuniculteurs professionnels utilisent une stratégie de restriction, pour améliorer l'état sanitaire de leur troupeau et pour réduire le coût alimentaire. Globalement, la marge faite sur le coût alimentaire est estimé à $0,30 €$ par lapin sevré. La consommation de médicaments (notamment antibiotiques) est estimée entre 0,10 et $0,15 €$ par lapin et on peut s'attendre à une réduction de ce coût grâce à la stratégie de restriction temporaire de l'ingestion. Toutefois, la rentabilité d'une stratégie de restriction variera selon le cours des aliments et le poids d'abattage des lapins.

Les effets bénéfiques d'une limitation de l'ingestion chez le lapin en croissance ont été démontrés dans diverses conditions expérimentales et de terrain. Nous pouvons recommander de limiter la consommation d'au moins $20 \%$ au cours des deux semaines suivant le sevrage, pour réduire les risques de troubles digestifs. Cependant, les stratégies de limitation de l'ingestion doivent être adaptées à chaque situation d'élevage et optimisées selon les objectifs du cuniculteur : améliorer l'état de santé, réduire le coût d'alimentation, stabiliser les performances. C'est pourquoi de nombreuses stratégies de restriction ont été développées en France pour s'adapter à la spécificité de chaque système d'élevage et à ses objectifs.

Les mécanismes physiologiques expliquant les effets favorables d'une ingestion restreinte sur la santé digestive du lapin restent à identifier. Une approche interdisciplinaire devra être utilisée pour explorer plus précisément la réponse physiologique du jeune lapin, par exemple en étudiant les relations entre la réponse inflammatoire, le statut immunitaire, la maturation du microbiote et le niveau d'ingestion. 


\section{Références}

Ben Rayana A., Ben Amouda M., Bergaoui R., 2008. Effect of water restriction times of 2 and $4 \mathrm{~h}$ per day on performances of growing rabbits. $9^{\text {th }}$ World Rabbit Congr., Xiccato G., Trocino A., Lukefahr S.D. (Eds). 10-13 june, Verona, Italy, 541-545.

Bergaoui R., Kammoun M., Ouerdiane K. 2008. Effects of feed restriction on the performance and carcass of growing rabbits. $9^{\text {th }}$ World Rabbit Congr., Xiccato G., Trocino A., Lukefahr S.D. (Eds). 10-13 june, Verona, Italy, 547-550.

Boisot P., Licois D., Gidenne T., 2003. Feed restriction reduces the sanitary impact of an experimental reproduction of Epizootic Rabbit Enteropathy syndrom (REE), in the growing rabbit. $10^{\text {emes }}$ Journ. Rech. Cunicoles, Paris, France, 267-270.

Boisot P., Duperray J., Dugenétais X., Guyonvarch A., 2004. Interest of hydric restriction times of 2 and $3 \mathrm{~h}$ per day to induce feed restriction in growing rabbits. $8^{\text {th }}$ World Rabbit Congr., Becerril C., Pro A. (Eds). Mexico, 759-764. http://www.dcam.upv.es/758wrc/

Boisot P., Duperray J., Guyonvarch A., 2005. Intérêt d'une restriction hydrique en comparaison au rationnement alimentaire en bonnes conditions sanitaires et lors d'une reproduction expérimentale de l'Entéropathie Epizootique du Lapin (EEL). $11^{\text {èmes }}$ Journ. Rech. Cunicoles, 29-30 novembre, Paris, France, 133-136.

Bovera F., Piccolo G., D’Urso S., Nizza S., Cutrignelli M.I., 2008. Feed restriction during summer: effect on rabbit carcass traits and meat quality. $9^{\text {th }}$ World Rabbit Congr., Xiccato G., Trocino A., Lukefahr S.D. (Eds). 10-13 june, Verona, Italy, 1325-1330.

Cantier J., Vezinhet A., Rouvier R., 1969. Allométrie de croissance chez le lapin. I) Principaux organes et tissus. Ann. Biol. Anim. Biochim. Biophys., 14, 271-292.

Carabaño R., Badiola I., Chamorro S., García J., García-Ruiz AI., García-Rebollar P., Gómez-Conde M.S., Gutiérrez I., Nicodemus N., Villamide M.J., De Blas J.C., 2008. New trends in rabbit feeding: influence of nutrition on intestinal health. Span. J. Agric. Res., 6, 15 25 .

Chevance A., Moulin G., 2009. Suivie des ventes de médicaments vétérinaires contenant des antibiotiques en France en 2008. Rapport d'étude, ANSES Publ., 43. http://www.afssa.fr

Dalle Zotte A., Ouhayoun J., 1998. Effect of genetic origin, diet and weaning weight on carcass composition, muscle physicochemical and histochemical traits in the rabbit. Meat Sci., 50, 471-478.

Dalle Zotte A., Rémignon H., Ouhayoun J., 2005. Effect of feed rationing during postweaning growth on meat quality, muscle energy metabolism and fibre properties of Biceps femoris muscle in the rabbit. Meat Sci., 70, 301-306.

Diaz Arca F., Perez Alba LM., Perez Hernandez M., 1999. Digestibility and energy retention by young rabbits fed different levels of intake. Ann. Zootech., 48, 289-295.

Di Meo C., Bovera F., Marono S., Vella N., Nizza A., 2007. Effect of feed restriction on performance and feed digestibility in rabbits. Italian J. Anim. Sci., 6, 765-767.
Duperray J., Gyonvarch A., 2009. Effect of different quantitative feed restriction strategies on fattening performance, and interest of a high energy and protein feed. $13^{\text {èmes }}$ Journ. Rech. Cunicoles, 17-18 novembre, Le Mans, France, 59-62.

Elmaghraby M.M.A., 2011. Effect of restricted access to drinking water on growth, feed efficiency and carcass characteristics of fattening rabbits. Asian J. Anim. Sci., 5, 136144.

Fioramonti J., Ruckebusch Y., 1974. La motricité caecale chez le lapin. 2) Variations d'origine alimentaire. Ann. Rech. Vet., 5, 201212.

Fodor K., Fekete S.G., Zoldag L., Bersenyi A., Gaspardy A., Andrasofszky E., Kulcsar M., Eszes F., 2001. Influence of feeding intensity on corporeal development, body composition and sexual maturity in female rabbits. Acta Vet. Hungarica, 49, 399-411.

Foubert C., Boisot P., Duperray J., Guyonvarch A., 2007. Interêt d'un accès limité à la mangeoire de $6 \mathrm{~h}, 8 \mathrm{~h}$ et $10 \mathrm{~h}$ par jour pour engendrer un rationnement alimentaire chez le lapin en engraissement. $12^{\text {èmes }}$ Journ. Rech. Cunicoles, 27-28 novembre, Le Mans, France, 123-126.

Foubert C., Duperray J., Guyonvarch A., 2008a. Intérêt d'un aliment fibreux concentré en énergie chez le lapin de chair rationné. Journ. Nationale ITAVI Elevage du lapin de chair, Pacé, 25 novembre, Paris, France, 1-8.

Foubert C., Duperray J., Boisot P. Guyonvarch A., 2008b. Effect of feed restriction with or without free access to drinking water on performance of growing rabbits in healthy or epizootic rabbit enteropathy conditions. $9^{\text {th }}$ World Rabbit Congr., Xiccato G., Trocino A., Lukefahr S.D. (Eds). 10-13 june, Verona, Italy, 667-671.

Gallois M., Gidenne T., Fortun-Lamothe L., Le Huerou-Luron I., 2005. An early stimulation of solid feed intake slightly influences the morphological gut maturation in the rabbit. Reprod. Nutr. Dev., 45, 109-122.

Gidenne T., 1987. Effet de l'addition d'un concentré riche en fibres dans une ration à base de foin, distribuée à deux niveaux alimentaires chez la lapine adulte. 2/ Mesures de digestibilité. Reprod. Nutr. Dev., 27, 801-810.

Gidenne T., 2003. Fibres in rabbit feeding for digestive troubles prevention: respective role of low-digested and digestible fibre. Livest. Prod. Sci., 81, 105-117.

Gidenne T., Bellier R., 1992. Etude in vivo de l'activité fermentaire caecale chez le lapin. Mise au point et validation d'une nouvelle technique de canulation caecale. Reprod. Nutr. Dev., 32, 365-376.

Gidenne T., Lebas F., 2006. Feeding behaviour in rabbits. In, Feeding in domestic vertebrates. From structure to behaviour. Bels V. (Ed). CABI publ., Wallingford, UK, 179-209.

Gidenne T., Feugier A., 2009. Feed restriction strategy in the growing rabbit. 1. Impact on digestion, rate of passage and microbial activity. Animal, 3, 501-508.

Gidenne T., Poncet C., Gomez L., 1987. Effet de l'addition d'un concentré riche en fibres dans une ration à base de foin, distribuée à deux niveaux alimentaires chez la lapine adulte. 1/ Temps de séjour moyen des aliments. Reprod. Nutr. Dev., 7, 733-743.

Gidenne T., Feugier A., Jehl N., Arveux P., Boisot P., Briens C., Corrent E., Fortune H., Montessuy S., Verdelhan S., 2003. Un rationnement alimentaire quantitatif post-sevrage permet de réduire la fréquence des diarrhées, sans dégradation importante des performances de croissance : résultats d'une étude multi-site. $10^{\text {èmes }}$ Journ. Rech. Cunicoles, 19-20 novembre, Paris, France, 29-32.

Gidenne T., Combes S., Feugier A., Jehl N., Arveux P., Boisot P., Briens C., Corrent E. Fortune H., Montessuy S., Verdelhan S., 2009a. Feed restriction strategy in the growing rabbit. 2. Impact on digestive health, growth and carcass characteristics. Animal, 3, 509515 .

Gidenne T., Murr S., Travel A., Corrent E., Foubert C., Bebin K., Mevel L., Rebours G., Renouf B., 2009b. Effets du niveau de rationnement et du mode de distribution de l'aliment sur les performances et les troubles digestifs post-sevrage du lapereau. Premiers résultats d'une étude concertée du réseau GEC. Cuniculture Magazine, 36, 65-72.

Gidenne T., Bannelier C., Combes S., Fortun-Lamothe L., 2009c. Interaction entre la stratégie de restriction et la concentration énergétique de l'aliment : impact sur la croissance et la santé du lapin. Premiers resultants. $13^{\text {èmes }}$ Journ. Rech. Cunicoles, 17-18 novembre, Le Mans, France, 63-66.

Gidenne T., Travel A., Murr S., Oliveira H., Corrent E., Foubert C., Bebin K., Mevel L., Rebours G., Renouf B., Gigaud V., 2009d. Ingestion restreinte et mode de distribution de la ration. Conséquences sur le comportement alimentaire, la digestion et la qualité de la carcasse. $13^{\text {emes }}$ Journ. Rech. Cunicoles, 22-23 novembre, Le Mans, France, 43-46.

Gidenne T., Garcia J., Lebas F., Licois D., 2010. Nutrition and feeding strategy: interactions with pathology. In: Nutrition of the rabbit. De Blas C., Wiseman J. (Eds). CABI publ., Wallingford, UK, 179-199.

Gidenne T., Combes S., Briens C., Duperray J., Rebours G., Salaun J.M., Weissman D. Fortun-Lamothe L., Combe Y., Travel A., 2011. Ingestion restreinte et concentration protéique de l'aliment: impact sur la digestion et les rejets azotés. $13^{\text {èmes }}$ Journ. Rech. Cunicoles, 22-23 novembre, Le Mans, France, 21-24.

Gidenne T., Combes S., Briens C., Duperray J., Mevel L., Rebours G., Salaun J.M., Weissman D., Combe Y., Travel A., 2012a. Intake limitation strategy and dietary protein concentration: effect on rabbit growth performance and health, from a large-scale study in a french network of experimental units (GEC). 10 ${ }^{\text {th }}$ World Rabbit Congr., 4-6 septembre, Sharm El Sheik, Egypt.

Gidenne T., Combes S., Fortun-Lamothe L., 2012 b. Feed intake limitation strategies for the growing rabbit: effect on feeding behaviour, welfare, performance, digestive physiology and health: a review. Animal, 6, 14071419.

Gondret F., Lebas F., Bonneau M., 2000. Restricted feed intake during fattening reduces intramuscular lipid deposition without modifying muscle fiber characteristics in rabbits. J. Nutr., 130, 228-233. 
Jérome N., Mousset J.L., Messager B. Deglaire I., Marie P., 1998. Influence de différentes méthodes de rationnement sur les performances de croissance et d'abattage du lapin. $7^{\text {èmes }}$ Journ. Rech. Cunicoles, Lyon, France, 175-178

Jolly C.A., 2004. Dietary restriction and immune function. J. Nutr., 134: 1853-1856.

Lebas F., 1979. Efficacité de la digestion chez la lapine adulte. Effet du niveau d'alimentation et du stade de gestation. Ann. Biol. Anim. Bioch. Biophys., 19, 969-973.

Lebas F., 2007. L'utilisation de la restriction alimentaire dans la filière cunicole. Cuniculture magazine. ASFC. http://www.asfclapin.com/Docs/Activite/T-ronde-2007/Tronde2007-1.htm

Lebas F., Delaveau A., 1975. Influence de la restriction du temps d'accès à la boisson sur la consommation alimentaire et la croissance du lapin. Ann. Zootech., 24, 311-313.

Lebas F., Laplace J.P., 1982. Mensurations viscérales chez le lapin. 4. Effets de divers modes de restriction alimentaire sur la croissance corporelle et viscérale. Ann. Zootech. 31, 391-430

Ledin I., 1984a. Effect of restricted feeding and realimentation on growth, carcass composition and organ growth during the first seven days of realimentation in rabbit. Acta Agric. Scand., 34, 54-66.

Ledin I., 1984b. Effect of restricted feeding and realimentation on compensatory growth and organ growth in rabbit. Ann. Zootech., 33 $33-50$

Maertens L., Peeters J.E., 1988. Effect of feed restriction after weaning on fattening performances and caecal traits of early weaned rabbits. Proc. of the Deutsche Veterinarmediznische Gesellschaft, Celle, Germany, 158-169.

Marlier D., Dewree R., Delleur V., Licois D., Lassence C., Poulipoulis A., Vindevogel H., 2003. A review of the major causes of digestive disorders in the European rabbit. Ann. Med. Vet., 147, 385-392.

Martignon M.H., 2010. Conséquences d'un contrôle de l'ingestion sur la physiopathologie digestive et le comportement alimentaire. $\mathrm{PhD}$ thesis, Institut National Polytechnique de Toulouse, France, 194p. http://ethesis.inp-toulouse.fr/archive/00001486/

Martignon M.H., Combes S., Gidenne T., 2009. Rôle du mode de distribution de 1'aliment dans une stratégie de rationnement conséquences sur le profil d'ingestion, la croissance et la santé digestive du lapin. $13^{\text {emes }}$ Journ. Rech. Cunicoles, Bolet G. (Ed). 17-18 novembre, Le Mans, France, 39-42.

Martignon M.H., Combes S., Gidenne T., 2010. Digestive physiology and hindgut bacterial community of the young rabbit (Oryctolagus cuniculus): Effects of age and short-term intake limitation. Comp. Bioch. Physiol. Part A, 156, 156-162.

Martignon M.H., Guinebretière M. Postollec G., Huonnic D., Boilletot E., Jourden A., Marie F., Burel C., Gidenne T., Michel V., 2011a. Conséquences d'une restriction alimentaire sur le comportement et le bien-être des lapins en engraissement. $13^{\text {èmes }}$ Journ. Rech. Cunicoles, 22-23 novembre, Le Mans, France, 25-28.

Martignon M.H., Licois D., Cauquil L., Amelot M., Combes S., Reperant E., Postollec
G., Gidenne T., Valat C., 2011b. Réponse digestive du lapereau à une inoculation expérimentale colibacillaire (O128:C6) en fonction du niveau d'ingestion. $13^{\text {emes }}$ Journ. Rech. Cunicoles, 22-23 novembre, Le Mans, France, 161-164.

Matics Z.S., Dalle Zotte A., Radnai I. Kovács M., Metzger S.Z., Szendrõ Z.S., 2008. Effect of restricted feeding after weaning on the productive and carcass traits of growing rabbits. $9^{\text {th }}$ World Rabbit Congr., Xiccato G., Trocino A., Lukefahr S.D. (Eds). 10-13 june, Verona, Italy, 741-745. http://world-rabbitscience.com/WRSA-Proc./Congress-2008Verona/Verona-2008-a.htm

Ouhayoun J., 1998. Influence of the diet on rabbit meat quality. In: The nutrition of the rabbit. De Blas C., Wiseman J. (Eds), CABI publ., Wallingford, UK, 177-195.

Palsson H., 1955. Conformation and body composition. In: Progress in the physiology of farm animals. Vol. II. Butterworth, London, UK, pp430.

Perrier G, 1998. Influence de deux niveaux et de deux durées de restriction alimentaire sur l'efficacité productive du lapin et les caractéristiques bouchères de la carcasse. $7^{\text {èmes }}$ Journ. Rech. Cunicoles, 13-14 mai, Lyon, France, 179-182.

Perrier G., Ouhayoun J., 1996. Growth and carcass traits of the rabbit a comparative study of three modes of feed rationing during fattening. $6^{\text {th }}$ World Rabbit Congr., Toulouse, France, 225-232.

Prud'hon M., Cherubin M., Goussopoulos J., Carles Y., 1975. Evolution au cours de la croissance des caractéristiques de la consommation d'aliments solides et liquides du lapin domestique nourri ad libitum. Ann. Zootech., 24 289-298.

Rebollar P.G., Pereda N., Schwarz B.F., Millán P., Lorenzo P.L., Nicodemus N., 2011. Effect of feed restriction or feeding high-fibre diet during the rearing period on body composition, serum parameters and productive performance of rabbit does. Anim. Feed Sci. Technol., 163, 67-76.

Romero C., Cuesta S., Astillero J.R. Nicodemus N., De Blas C., 2010. Effect of early feed restriction on performance and health status in growing rabbits slaughtered at $2 \mathrm{~kg}$ live-weight. World Rabbit Sci., 18, 211-218.

Romero C., Rebollar P.G., Bosco A.D., Castellini C., Cardinali R., 2011. Dietary effect of short-chain organic acids on growth performance and development of intestinal lymphoid tissues in fattening restricted rabbits. World Rabbit Sci., 19: 133-142.

Ruckebusch Y., Grivel M.L., Fargeas M.J., 1971. Activité électrique de l'intestin et prise de nourriture conditionnelle chez le lapin. Physiol. Behav., 6, 359-366.

Schlolaut W., Lange K., Schluter H., 1978. Influence de l'intensite alimentaire sur les performances d'engraissement et la qualité de carcasse chez le lapereau à l'engrais. Zuchtungskunde, 50, 401-411.

Szendrő Z., Szabo S., Hullar I., 1988. Effect of reduction of eating time on production of growing rabbits. World Rabbit Congr. Budapest, Hungary, 104-114

Szendrő Z., Metzger S., Fébel H., Hullár I. Maertens L., Bianchi M., Cavani C., Petracci M., Biró-Németh E., Radnai I., 2008. Effect of energy restriction in interaction with genotype on the performance of growing rabbits I: Productive traits. Livest. Sci., 118, 123-131.

Taranto S., Di Meo C., Stanco G., Piccolo G., Gazaneo MP., Nizza A., 2003. Influence of age at weaning on caecal content characteristics and post-weaning performance and health of rabbits. Asian-Australasian J. Anim. Sci., $16,1540-1544$

Travel A., Briens C., Duperray J., Mevel L., Rebours G., Salaun J.M., Weissman D., Combe Y., Gidenne T., 2011. Ingestion restreinte et concentration protéique de l'aliment: Impact sur le rendement carcasse et la qualité de la viande de lapins. $13^{\text {èmes }}$ Journ. Rech. Cunicoles, 22-23 novembre, Le Mans, France, 105-109.

Tudela F., Lebas F., 2006. Modalités du rationnement des lapins en engraissement. Effets du mode de distribution de la ration quotidienne sur la vitesse de croissance, le comportement alimentaire et l'homogénéité des poids. Cuniculture Magazine, 33, 21-27. ht t p : / / w w w. cun iculture.info/ Docs/Magazine/Magazine2006/mag33$\underline{021 . h t m}$

Tumova E., Skrivan M., Skrivanova V., Kacerivska L., 2002. Effect of early feed restriction on growth in broiler chickens, turkeys and rabbits. Czech J. Anim. Sci., 47, 418-428.

Tumova E., Skrivanova V., Skrivan M. 2003. Effect of restricted feeding time and quantitative restriction in growing rabbits. Archiv Fur Geflügelk., 67, 182-190.

Tumova E., Zita L., Stolc L., 2006. Carcass quality in restricted and ad libitum fed rabbits. Czech J. Anim. Sci., 51, 214-219.

Tumova E., Zita L., Skrivanova V., Fucikova A., Skrivan M., Buresova M., 2007. Digestibility of nutrients, organ development and blood picture in restricted and ad libitum fed broiler rabbits. Archiv Fur Geflügelkunde, 71, 6-12.

Van Harten S., Cardoso L.A., 2010. Feed restriction and genetic selection on the expression and activity of metabolism regulatory enzymes in rabbits. Animal, 4, 1873-1883.

Verdelhan S., Bourdillon A., Morel-Saives A., Audoin E., 2004. Effect of a limited access to water on mortality of fattening rabbits. $8^{\text {th }}$ World Rabbit Congr., Becerril C., Pro A. (Eds). 7-10 sept., Puebla, Mexico, Colegio de Post-graduados for WRSA publ., 10151021. http://www.dcam.upv.es/1018wrc/

Weissman D., Troislouches G., Picard E. Davoust C., Leroux C., Launay C., 2009. Amélioration de l'indice de consommation de lapins en engraissement par une distribution nocture de 1'aliment. $13^{\text {èmes }}$ Journ. Rech. Cunicoles, 17-18 novembre, Le Mans, France, $1-3$.

Xiccato G., Cinetto M., 1988. Effect of nutritive level and age on feed digestibility and nitrogen balance in rabbit. $4^{\text {th }}$ World Rabbit Congr., Budapest, Hungary, 96-103.

Xiccato G., Cinetto M., Dalle Zotte A. 1992. Effect of feeding plane and category of rabbit on digestive efficiency and nitrogen balance. Zootec Nutr. Anim., 181, 35-43.

Yakubu A., Salako A.E., Ladokun A.O. Adua M.M., Bature T.U.K., 2007. Effects of feed restriction on performance, carcass yield, relative organ weights and some linear body measurements of weaner rabbits. Pakistan J. Nutr., 6, 391-396. 


\title{
Résumé
}

La restriction temporaire de l'ingestion du lapin après son sevrage est maintenant une stratégie d'alimentation couramment employée en cuniculture. L'objet de cette synthèse est de présenter les différents effets d'une limitation de l'ingestion sur la santé, la physiologie digestive et l'efficacité alimentaire du lapin en croissance. Même si une restriction alimentaire conduit à une croissance plus lente, ces stratégies sont désormais utilisées par plus de $85 \%$ des éleveurs français. En effet, elles permettent de réduire les risques de mortalité et de morbidité post-sevrage par troubles digestifs, par exemple par l'entéropathie épizootique du lapin. De plus, la conversion alimentaire est améliorée, plus particulièrement lorsque les lapins sont de nouveau alimentés librement, en raison d'une importante croissance compensatrice. Cette meilleure efficacité alimentaire est associée à un transit plus lent et à une meilleure digestion, bien qu'on observe des interactions avec la composition chimique de l'aliment. La physiologie digestive est par ailleurs peu modifiée, en particulier la morphométrie de la muqueuse intestinale, l'activité fermentaire et le microbiote caecal. La qualité de la viande est peu affectée par la restriction alimentaire, alors qu'on observe une baisse de l'état d'engraissement des carcasses et une légère dégradation du rendement à l'abattage. Les effets d'une limitation de l'ingestion sur le comportement et le bien-être animal sont discutés, sachant que le jeune lapin présente une adaptation très rapide aux stratégies de restriction. Ainsi, les stratégies de restriction peuvent améliorer la rentabilité de l'atelier cunicole, mais elles doivent être adaptées à chaque situation d'élevage.

\begin{abstract}
Intake restriction for the young rabbit: new strategies to enhance its digestive health and feed efficiency

A short-term feed restriction after weaning is now a feeding strategy commonly used in rational rabbit breeding. The different effects produced by a post-weaning intake limitation strategy on the growing rabbit are reviewed. Although a quantitative feed restriction leads to slower growth, these strategies are now used by $90 \%$ of French rabbit breeders. Indeed, after weaning, they limit the risk of mortality and morbidity due to digestive disorders (e.g. Rabbit Epizootic Enteropathy). In addition, feed conversion is improved, especially when rabbits are fed freely again, due to a significant compensatory growth. This better feed conversion sources from a better digestion associated with a slower passage through the intestine, whereas the digestive physiology is slightly modified (morphometry of the intestinal mucosa, fermentation pattern, microbiota). The meat quality is unaffected by feed restriction, however there is a decrease in carcass fatness and a slight degradation in slaughter yield. The consequences of an intake limitation for animal welfare are debatable, since feed restriction leads to hunger, but it reduces the incidence of digestive troubles after weaning. However, the growing rabbit adapts very well to an intake limitation strategy, without any aggressive behaviour within a group. In conclusion, restriction strategies could improve profitability of rabbit breeding, but they should be adapted to any specific breeding situation, according to the national market, feed prices, etc.
\end{abstract}

GIDENNE T., FORTUN-LAMOTHE L., COMBES S., 2012. Restreindre l'ingestion du jeune lapin : de nouvelles stratégies pour renforcer sa santé digestive et améliorer son efficacité alimentaire. INRA Prod. Anim., 25, 4, 323336. 